\title{
$B V$ functions and parabolic initial boundary value problems on domains
}

\author{
Luciana Angiuli • Michele Miranda Jr • \\ Diego Pallara • Fabio Paronetto
}

Received: 23 May 2007 / Revised: 28 November 2007 / Published online: 25 April 2008

C Springer-Verlag 2008

\begin{abstract}
Given a uniformly elliptic second order operator $\mathcal{A}$ on a possibly unbounded domain $\Omega \subset \mathbb{R}^{N}$, let $(T(t))_{t \geq 0}$ be the semigroup generated by $\mathcal{A}$ in $L^{1}(\Omega)$, under homogeneous co-normal boundary conditions on $\partial \Omega$. We show that the limit as $t \rightarrow 0$ of the $L^{1}$-norm of the spatial gradient $D_{x} T(t) u_{0}$ tends to the total variation of the initial datum $u_{0}$, and in particular is finite if and only if $u_{0}$ belongs to $B V(\Omega)$. This result is true also for weighted $B V$ spaces. A further characterization of $B V$ functions in terms of the short-time behaviour of $(T(t))_{t \geq 0}$ is also given.
\end{abstract}

Keywords Linear parabolic equations $\cdot B V$ functions $\cdot$ Semigroup theory

Mathematics Subject Classification (2000) $\quad 35 \mathrm{~K} 20 \cdot 47 \mathrm{D} 06 \cdot 49 \mathrm{Q} 15$

\section{Introduction}

The definition of functions with bounded variation in $\mathbb{R}^{N}, N \geq 2$, has been given by De Giorgi [9] using the Gauss-Weierstrass kernel $g_{t}(x)=(4 \pi t)^{-N / 2} e^{-|x|^{2} / 4 t}$ : given $u \in L^{1}\left(\mathbb{R}^{N}\right)$, he

\footnotetext{
L. Angiuli · D. Pallara · F. Paronetto $(\varangle)$

Dipartimento di Matematica "Ennio De Giorgi”, Università del Salento,

P. O. Box 193, 73100 Lecce, Italy

e-mail: fabio.paronetto@unile.it

L. Angiuli

e-mail: luciana.angiuli@unile.it

D. Pallara

e-mail: diego.pallara@unile.it

M. Miranda Jr

Dipartimento di Matematica, Università di Ferrara,

Via Machiavelli, 35, 44100 Ferrara, Italy

e-mail: michele.miranda@unife.it
} 
defined the total variation of $u$ by

$$
|D u|\left(\mathbb{R}^{N}\right)=\lim _{t \rightarrow 0} \int_{\mathbb{R}^{N}}\left|D\left(u * g_{t}\right)\right| d x,
$$

where it is easily seen that the integral on the right hand side is a monotone function in $t$, and then the limit exists. Notice that $g_{t}$ is the heat kernel on $\mathbb{R}^{N}$, and then, using the language of semigroups and setting $T(t) u=u * g_{t}$, formula (1.1) can be written as

$$
|D u|\left(\mathbb{R}^{N}\right)=\lim _{t \rightarrow 0} \int_{\mathbb{R}^{N}}|D T(t) u| d x .
$$

Moreover, the fact that $|D u|\left(\mathbb{R}^{N}\right)$ is finite is equivalent to saying that the distributional gradient of $u$ is a ( $\mathbb{R}^{N}$-valued) Radon measure, and this gives the equality

$$
|D u|\left(\mathbb{R}^{N}\right)=\sup \left\{\int_{\mathbb{R}^{N}} u \operatorname{div} \phi d x: \phi \in C_{c}^{1}\left(\mathbb{R}^{N}, \mathbb{R}^{N}\right),\|\phi\|_{L^{\infty}\left(\mathbb{R}^{N}\right)} \leq 1\right\} .
$$

A formula analogous to (1.2) can also be used in more general contexts. The question then arises if the connection between semigroups and total variation is more general; recently, in $[6,16]$ this connection has been investigated in the setting of Riemannian manifolds with a bound on the geometry. Formula (1.3) can be localized in a subset $\Omega \subset \mathbb{R}^{N}$ by

$$
|D u|(\Omega)=\sup \left\{\int_{\Omega} u \operatorname{div} \phi d x: \phi \in C_{c}^{1}\left(\Omega, \mathbb{R}^{N}\right),\|\phi\|_{L^{\infty}(\Omega)} \leq 1\right\}
$$

and gives the definition of total variation of $u \in L^{1}(\Omega)$.

In this paper we prove that (1.2) still holds in $\Omega$, when $(T(t))_{t \geq 0}$ is the semigroup generated by a second order uniformly elliptic operator with regular coefficients and suitable boundary conditions. Concerning the monotonicity, in $\mathbb{R}^{N}$ the inequality

$$
\int_{\mathbb{R}^{N}}|D T(t) u| d x \leq|D u|\left(\mathbb{R}^{N}\right), \quad \forall t>0,
$$

holds, whereas in the Riemannian case the following is true:

$$
\int_{M}|D T(t) u| d \mu \leq e^{k t}|D u|(M), \quad \forall t>0,
$$

where $k>0$ is a constant bounding the geometry of $M$. Here we consider the uniformly elliptic operator with sufficiently smooth coefficients

$$
\mathcal{A} u=\sum_{i, j=1}^{N} D_{i}\left(A_{i j} D_{j} u\right)+\sum_{i=1}^{N} B_{i} D_{i} u+C u
$$

and the initial-boundary value problem

$$
\begin{cases}\partial_{t} w-\mathcal{A} w=0 & \text { in }(0, \infty) \times \Omega \\ w(0)=u_{0} & \text { in } \Omega \\ \langle A D w, v\rangle=0 & \text { in }(0, \infty) \times \partial \Omega\end{cases}
$$


where $v$ is the outward unit normal. Denoting again by

$$
T(t) u_{0}(x)=\int_{\Omega} p(t, x, y) u_{0}(y) d y
$$

the semigroup which gives its solution, we prove that the inequality

$$
\int_{\Omega}|D T(t) u|_{A} d x \leq|D u|_{A}(\Omega)+c\|u\|_{W^{1,1}(\Omega)} t^{\gamma}, \quad t \in(0,1),
$$

holds for $u$ in the domain of $\mathcal{A}$ (see (4.1) for the definition of the weighted total variation) and $0<\gamma<1 / 2$, and deduce (1.2) by approximating $u \in B V(\Omega)$ in variation, and taking the limit as $t \rightarrow 0$. We point out that equality (1.2) holds not only for classical $B V$ functions, but also for weighted $B V$ functions, see Theorem 5.2.

A further characterization of $B V$ functions can be given considering in a different way the short-time behaviour of $(T(t))_{t \geq 0}$, namely, we prove that

$$
|D u|_{A}(\Omega)=\lim _{t \rightarrow 0} \frac{\sqrt{\pi}}{2 \sqrt{t}} \int_{\Omega} \int_{\Omega} p(t, x, y)|u(x)-u(y)| d y d x,
$$

where $|D u|_{A}$ denotes the $\left(A_{i j}\right)$-weighted total variation of $u$. This characterization is in the spirit of $[4,8,13,15]$, where kernels depending on $|x-y|$ are considered.

The paper is organized as follows; after recalling some preliminary results in Sect. 2, we discuss in Sect. 3 the main properties of the semigroup associated with second order partial differential operator on unbounded domains. Using these results, and after giving the definitions and main properties of weighted $B V$ functions in Sect. 4, we prove in Sect. 5 the limiting formula (1.2). Sect. 6 is devoted to the proof of (1.5).

\section{Notations and preliminary results}

We denote by $\langle\cdot, \cdot\rangle$ the standard inner product on $\mathbb{R}^{N}$, by $|\cdot|$ its induced norm and by $B_{\varrho}(x)$ the open ball centred at $x$ and with radius $\varrho$. Moreover, given a symmetric positive definite matrix $P$ we introduce the norm

$$
|\xi|_{P}^{2}=\left|P^{1 / 2} \xi\right|^{2}=\langle P \xi, \xi\rangle, \quad \forall \xi \in \mathbb{R}^{N}
$$

we use the same notation even for variable matrices $P$. By $C_{b}(\Omega)$ we mean the set of continuous and bounded functions on $\Omega$, by $C_{c}(\Omega)$ the set of function $u$ with support strictly contained in $\Omega$, that is $\operatorname{supp} u \subset \subset \Omega$ and by $C_{c}(\bar{\Omega})$ the set of functions with support a compact set contained in $\bar{\Omega}$ (then not necessarily zero on $\partial \Omega$ ). For functions $u \in C_{b}^{k}(\Omega)$ we define the norms $\|u\|_{\infty}=\|u\|_{L^{\infty}(\Omega)},\|\cdot\|_{k, \infty}=\sum_{|\alpha| \leq k}\left\|D^{\alpha} \cdot\right\|_{\infty}$. Moreover, given a matrix $Q$, we define

$$
C_{Q}(\Omega)=\left\{u \in C^{\infty}(\Omega) \cap C^{1}(\bar{\Omega}) ;\langle Q D u, v\rangle=0 \text { on } \partial \Omega\right\} .
$$

Given a subset $E \subset \mathbb{R}^{N}$, we denote by $|E|$ its Lebesgue measure, and by $\mathcal{H}^{N-1}(E)$ its $(N-1)$-dimensional Hausdorff measure. By $L^{p}(\Omega)(p \geq 1)$ we denote the Lebesgue space of $p$-integrable function with respect to the Lebesgue measure and by $W^{k, p}(\Omega)$ the space of functions $p$-integrable together with their distributional derivatives up to the $k$ th order. We recall that if the open set $\Omega$ has regular boundary, then the trace operator is continuous 
from $W^{1,1}(\Omega)$ onto $L^{1}\left(\partial \Omega, \mathcal{H}^{N-1}\right)$ (see for instance [1, Theorem 5.3.6]), that, is there exists $c_{\Omega}>0$ such that for every $u \in W^{1,1}(\Omega)$ the trace $v=u_{\mid \partial \Omega}$ of $u$ on $\partial \Omega$ is well defined and

$$
\|v\|_{L^{1}\left(\partial \Omega, \mathcal{H}^{N-1}\right)} \leq c_{\Omega}\|u\|_{W^{1,1}(\Omega)} .
$$

In the whole paper we denote by $\Omega$ an open subset of $\mathbb{R}^{N}$, not necessarily bounded, and we always assume that $\Omega$ has uniformly $C^{2}$ boundary, that is, there are $\varrho, L>0$ such that for every $x \in \partial \Omega$ the set $\partial \Omega \cap B_{\varrho}(x)$ is the graph of a $C^{2}$ function $\psi$ with $\left\|D^{2} \psi\right\|_{2, \infty}$, $\left\|D^{2} \psi^{-1}\right\|_{2, \infty} \leq L$.

We consider the second order differential operator in divergence form

$$
\mathcal{A} u=\operatorname{div}(A D u)+\langle B, D u\rangle+C u=\sum_{i, j=1}^{N} D_{i}\left(A_{i j} D_{j} u\right)+\sum_{i=1}^{N} B_{i} D_{i} u+C u
$$

and the first order operator (co-normal derivative) on $\partial \Omega$

$$
\mathcal{B} u=\langle A D u, v\rangle=\sum_{i, j=1}^{N} A_{i j} D_{j} u v_{i}
$$

where $v$ is the outward unit normal to $\partial \Omega$. We say that a symmetric positive definite matrix $A$ is $\lambda$-elliptic if there exists $\lambda \geq 1$ such that $\lambda^{-1}|\xi|^{2} \leq\langle A \xi, \xi\rangle \leq \lambda|\xi|^{2}$ for all $\xi \in \mathbb{R}^{N}$. In general, we say that $A$ is elliptic if it is $\lambda$-elliptic for some $\lambda \geq 1$. The operator $\mathcal{A}$ is said to be elliptic or $\lambda$-elliptic if the matrix $A$ is so. Let us state our standing hypotheses:

(H1) $\Omega \subset \mathbb{R}^{N}$ has uniformly $C^{2}$ boundary;

(H2) $\mathcal{A}$ is $\lambda$-elliptic for some $\lambda \geq 1$;

(H3) $A_{i j} \in C_{b}^{1}(\bar{\Omega}), B, C \in L^{\infty}(\Omega)$.

Under assumption (H3) it is then possible to define the finite quantity

$$
M_{0}=\max _{i, j}\left\{\left\|A_{i j}\right\|_{1, \infty},\left\|B_{i}\right\|_{\infty},\|C\|_{\infty}\right\} .
$$

We are interested in the realization of the operator $\mathcal{A}$ with boundary condition $\mathcal{B}$ in $L^{1}(\Omega)$; we denote by $D(\mathcal{A})$ the domain of such a realization, which is defined as the closure, in the graph norm, of $\left\{u \in C^{2}(\bar{\Omega}) \cap L^{1}(\Omega): \mathcal{A} u \in L^{1}(\Omega), \mathcal{B} u=0\right\}$; we also have that $C_{A}(\Omega)$ is a core. We recall in the next section that $(\mathcal{A}, D(\mathcal{A}))$ is sectorial and generates an analytic semigroup in $L^{1}(\Omega)$. After recalling some known properties of the semigroup and its integral kernel $p$, we show further estimates needed in the sequel. Finally, we use the notation $c_{n}=c(x, y, \ldots)$ with the meaning that the constant $c_{n}$ depends upon the quantities $x, y$, etc.

\section{Analytic semigroups in $L^{1}(\Omega)$ generated by elliptic operators}

In this section we recall the main properties of the operator $(\mathcal{A}, D(\mathcal{A}))$ and of the semigroup generated in $L^{1}(\Omega)$, and derive further estimates needed in the sequel. We collect the known results in the following statement. To simplify the statements, in this section we assume $C \leq 0$, so that the semigroup is contractive. This assumption is not restrictive for our purposes, as we may replace $\mathcal{A}$ by $\mathcal{A}-\omega$ for a suitable $\omega$, getting a contractive semigroup $\left(T^{\omega}(t)\right)_{t \geq 0}$ whose kernel is nothing but $p_{\omega}(t, x, y)=e^{\omega t} p(t, x, y)$. 
Theorem 3.1 Let $\Omega, \mathcal{A}, \mathcal{B}, D(\mathcal{A})$ be as specified in Sect. 2. Then, $(\mathcal{A}, D(\mathcal{A}))$ is sectorial and generates an analytic semigroup of contractions $(T(t))_{t \geq 0}$ in $L^{1}(\Omega)$; for the kernel $p:(0,+\infty) \times \Omega \times \Omega \rightarrow \mathbb{R}$ of the semigroup $(T(t))_{t \geq 0}$ the following estimates hold; there exist $b, c_{1}>0$ such that for $|\alpha|,|\beta|<2, x, y \in \Omega$ and $t>0$

$$
\left|D_{x}^{\alpha} D_{y}^{\beta} p(t, x, y)\right| \leq \frac{c_{1}}{t^{\frac{N+|\alpha|+|\beta|}{2}}} e^{-b \frac{|x-y|^{2}}{t}} .
$$

In addition, there are constants $c_{i}=c\left(\Omega, \lambda, M_{0}\right)>0, i=2,3$ such that, since the operator is sectorial, the following holds:

$$
\|T(t)\|_{\mathcal{L}\left(L^{1}(\Omega)\right)} \leq 1, \quad \sqrt{t}\|D T(t)\|_{\mathcal{L}\left(L^{1}(\Omega)\right)} \leq c_{2}, \quad t\|\mathcal{A} T(t)\|_{\mathcal{L}\left(L^{1}(\Omega)\right)} \leq c_{3},
$$

for $t>0$. Moreover, $D(\mathcal{A})$ is continuously embedded in $W^{1,1}(\Omega)$, i.e., there exists $c_{4}=$ $c\left(\Omega, \lambda, M_{0}\right)>0$ such that $v \in D(\mathcal{A})$ implies $v \in W^{1,1}(\Omega)$ and

$$
\|v\|_{W^{1,1}(\Omega)} \leq c_{4}\left(\|v\|_{L^{1}(\Omega)}+\|\mathcal{A} v\|_{L^{1}(\Omega)}\right) .
$$

Finally,

$$
\lim _{t \rightarrow 0}\|T(t) v-v\|_{W^{1,1}(\Omega)}=0
$$

for every $v \in D(\mathcal{A})$.

Proof Estimates (3.1) are proved in [18, Theorem 5.7]; from these, estimates (3.2) and (3.3) follow. To prove (3.4), if $v \in D(\mathcal{A})$, then $T(t) \mathcal{A} v=\mathcal{A} T(t) v$ and by the strong continuity of $T(t)$ in $L^{1}(\Omega)$ we get

$$
\begin{aligned}
\|D T(t) v-D v\|_{L^{1}(\Omega)} & \leq c_{4}\left(\|T(t) v-v\|_{L^{1}(\Omega)}+\|\mathcal{A} T(t) v-\mathcal{A} v\|_{L^{1}(\Omega)}\right) \\
& =c_{4}\left(\|T(t) v-v\|_{L^{1}(\Omega)}+\|T(t) \mathcal{A} v-\mathcal{A} v\|_{L^{1}(\Omega)}\right)
\end{aligned}
$$

and the statement is proved.

Remark 3.2 [Neumann boundary conditions] We have stated Theorem 3.1 in the form we most frequently use, but the estimates stated are known to hold under more general assumptions. In particular, all non-tangential boundary conditions are allowed. We denote by $c_{v}$ a constant which can be used in the first two inequalities in (3.2), when Neumann boundary conditions are associated with a general uniformly elliptic operator.

In order to proceed, we also need a precise $L^{1}$-estimate of the second (spatial) derivatives of $T(t) u_{0}$, for $u_{0} \in W^{1,1}(\Omega)$. This is proved in Proposition 3.5 below. The argument used here is similar to the one used in [10, Theorem 2.4], where $\Omega$ is bounded and different boundary conditions are imposed. The scheme is the following; we give estimates of the second order derivatives in Proposition 3.3, then, using this result, we characterize the interpolation space $D_{\mathcal{A}}(\alpha, 1)=\left(L^{1}(\Omega), D(\mathcal{A})\right)_{\alpha, 1}$ as a fractional Sobolev space and use this to improve estimate (3.5) using the $W^{1,1}$ norm of $u$ instead of the $L^{1}$ norm. We start with the following result.

Proposition 3.3 Let $\Omega, \mathcal{A}, \mathcal{B}$ be as in Sect. 2. Assume, in addition, $A \in W^{2, \infty}(\Omega)$ and $B, C \in W^{1, \infty}(\Omega)$; then, there exists $c_{5}$ depending on $N, \lambda, \Omega,\|A\|_{2, \infty},\|B\|_{1, \infty},\|C\|_{1, \infty}$, $c_{1}, c_{2}, c_{3} c_{v}$ such that for every $t \in(0,1)$ and $u \in L^{1}(\Omega)$ we have

$$
t\left\|D^{2} T(t) u\right\|_{L^{1}(\Omega)} \leq c_{5}\|u\|_{L^{1}(\Omega)} .
$$


Proof We set for $\sigma>0 u_{\sigma}=T(\sigma) u$ and $M_{1}=\max \left\{\|A\|_{2, \infty},\|B\|_{1, \infty},\|C\|_{1, \infty}\right\}$. By the regularity of the boundary $\partial \Omega$ we can consider a partition of unity $\left\{\left(\eta_{h}, U_{h}\right)\right\}_{h \in \mathbb{N}}$ such that supp $\eta_{h} \subset U_{h}, \sum_{h=0}^{\infty} \eta_{h}(x)=1$ for every $x \in \bar{\Omega}$ and $0 \leq \eta_{h} \leq 1$ for every $h \in \mathbb{N}, \bar{U}_{0} \subset \Omega$, $U_{h}$ for $h \geq 1$ is a ball such that $\left\{U_{h}\right\}_{h \geq 1}$ is a covering of $\partial \Omega$ and $\left\{U_{h}\right\}_{h \in \mathbb{N}}$ is a covering of $\Omega$ with bounded overlapping, that is there is $\kappa>0$ such that

$$
\sum_{h \in \mathbb{N}} \chi_{U_{h}}(x) \leq \kappa, \quad \forall x \in \bar{\Omega} .
$$

Moreover we choose $\eta_{h}$ in such a way that $\left\langle A(x) D \eta_{h}(x), v(x)\right\rangle=0$ for every $x \in \partial \Omega$ and set $M:=\sup _{h \in \mathbb{N}}\left\|\eta_{h}\right\|_{2, \infty}$, which is finite by the uniform $C^{2}$ regularity of $\partial \Omega$. Notice that $u_{\sigma} \in W^{1,1}(\Omega)$ and denote by $u(t)=T(t) u_{\sigma}$ the solution of the problem

$$
\begin{cases}\partial_{t} w-\mathcal{A} w=0 & \text { in }(0, \infty) \times \Omega, \\ w(0)=u_{\sigma} & \text { in } \Omega, \\ \langle A D w, v\rangle=0 & \text { in }(0, \infty) \times \partial \Omega .\end{cases}
$$

We want to estimate the $L^{1}$-norm of $t D^{2} u(t)$ by the $L^{1}$-norm of $u$; we shall use estimates (3.2). The functions $v_{h}(t)=u(t) \eta_{h}$ solve, for every $h \in \mathbb{N}$, the problem

$$
\begin{cases}\partial_{t} w-\mathcal{A} w=\mathcal{A}_{h} u(t) & \text { in }(0, \infty) \times \Omega, \\ w(0)=\eta_{h} u_{\sigma} & \text { in } \Omega, \\ \langle A D w, v\rangle=0 & \text { in }(0, \infty) \times \partial \Omega,\end{cases}
$$

where

$$
\mathcal{A}_{h} u(t)=-2\left\langle A D \eta_{h}, D u(t)\right\rangle-u(t) \operatorname{div}\left(A D \eta_{h}\right)-u(t)\left\langle B, D \eta_{h}\right\rangle .
$$

Notice that the derivative $D_{k} v_{h}(t)$ satisfies the equation $\partial_{t}\left(D_{k} v_{h}(t)\right)-\mathcal{A}\left(D_{k} v_{h}(t)\right)=\mathcal{A}_{h}^{k} u(t)$, where

$$
\begin{aligned}
\mathcal{A}_{h}^{k} u(t)= & \operatorname{div}\left(\left(D_{k} A\right) D\left(u(t) \eta_{h}\right)\right)+\left\langle\left(D_{k} B\right), D\left(u(t) \eta_{h}\right)\right\rangle+\left(D_{k} C\right) u(t) \eta_{h}+D_{k}\left(\mathcal{A}_{h} u(t)\right) \\
= & \operatorname{div}\left(\left(D_{k} A\right) D\left(u(t) \eta_{h}\right)\right)+\left\langle\left(D_{k} B\right), D\left(u(t) \eta_{h}\right)\right\rangle+\left(D_{k} C\right) u(t) \eta_{h} \\
& +D_{k}\left[-2\left\langle A D \eta_{h}, D u(t)\right\rangle-u(t) \operatorname{div}\left(A D \eta_{h}\right)-u(t)\left\langle B, D \eta_{h}\right\rangle\right] .
\end{aligned}
$$

For $D_{k} v_{h}(t)$ we consider the problem

$$
\begin{cases}\partial_{t} w-\mathcal{A} w=\mathcal{A}_{h}^{k} u(t) & \text { in }(0, \infty) \times \Omega, \\ w(0)=D_{k}\left(\eta_{h} u_{\sigma}\right) & \text { in } \Omega, \\ \langle A D w, v\rangle=0 & \text { in }(0, \infty) \times \partial \Omega\end{cases}
$$

whose solution is $v_{h k}(t)=T(t) D_{k}\left(\eta_{h} u_{\sigma}\right)+\int_{0}^{t} T(t-s) \mathcal{A}_{h}^{k} u(s) d s$. Now we consider $h=0$, i.e., we draw our attention to the inner part. Since $v_{0}(t)=\eta_{0} u(t)=0$ in $\Omega \backslash U_{0}$, it turns out that $D_{k} v_{0}(t)$ is the solution of (3.11) with $h=0$. Then

$$
D_{k} v_{0}(t)=T(t) D_{k}\left(\eta_{0} u_{\sigma}\right)+\int_{0}^{t} T(t-s) \mathcal{A}_{0}^{k} u(s) d s,
$$

where $\mathcal{A}_{0}^{k}$ is the operator defined in (3.10). Then, differentiating, we obtain

$$
D_{l k}^{2} v_{0}(t)=D_{l}\left[T(t) D_{k}\left(\eta_{0} u_{\sigma}\right)\right]+\int_{0}^{t} D_{l}\left[T(t-s) \mathcal{A}_{0}^{k} v(s)\right] d s
$$


by which, using (3.2),

$$
\begin{aligned}
\left\|D_{l k}^{2} v_{0}(t)\right\|_{L^{1}(\Omega)} & \leq\left\|D_{l} T(t) D_{k}\left(\eta_{0} u_{\sigma}\right)\right\|_{L^{1}(\Omega)}+\int_{0}^{t}\left\|D_{l} T(t-s) \mathcal{A}_{0}^{k} u(s)\right\|_{L^{1}(\Omega)} d s \\
& \leq \frac{c_{2}}{\sqrt{t}}\left\|D_{k}\left(\eta_{0} u_{\sigma}\right)\right\|_{L^{1}(\Omega)}+\int_{0}^{t} \frac{c_{2}}{\sqrt{t-s}}\left\|\mathcal{A}_{0}^{k} u(s)\right\|_{L^{1}(\Omega)} d s \\
& \leq \frac{c_{2}^{2}}{\sqrt{t \sigma}}\left\|\eta_{0}\right\|_{W^{1, \infty}}\|u\|_{L^{1}(\Omega)}+\int_{0}^{t} \frac{c_{2}}{\sqrt{t-s}}\left\|\mathcal{A}_{0}^{k} u(s)\right\|_{L^{1}(\Omega)} d s .
\end{aligned}
$$

Finally, estimating $\left\|\mathcal{A}_{0}^{k} u(s)\right\|_{L^{1}(\Omega)}$ by (3.10) we get $\left\|\mathcal{A}_{0}^{k} u(s)\right\|_{L^{1}(\Omega)} \leq c\|u(s)\|_{W^{2,1}(\Omega)}$ where $c=c\left(M, M_{1}\right)$. Summing on $l$ and $k$ and using again (3.2), we get

$$
\left\|D^{2} v_{0}(t)\right\|_{L^{1}(\Omega)} \leq c\left(\frac{1}{\sqrt{t \sigma}}\|u\|_{L^{1}(\Omega)}+\int_{0}^{t} \frac{1}{\sqrt{t-s}}\left\|D^{2} u(s)\right\|_{L^{1}(\Omega)} d s\right),
$$

where $c=c\left(M, M_{1}, c_{2}, N\right)$. We now consider $h \geq 1$, i.e., we consider a ball intersecting $\partial \Omega$. By the uniform $C^{2}$ regularity of $\partial \Omega$, we can consider coordinate functions $\psi_{h}: V_{h} \rightarrow B_{1}(0)$ such that $\psi_{h}\left(V_{h} \cap \Omega\right)=B_{1}^{-}(0)=\left\{y=\left(y^{\prime}, y_{N}\right) \in B_{1}(0): y_{N}<0\right\}, \psi_{h}\left(V_{h} \cap \partial \Omega\right)=\{y=$ $\left.\left(y^{\prime}, y_{N}\right) \in B_{1}(0): y_{N}=0\right\}$ and $d\left(\psi_{h}\right)_{x}(A(x) v(x))=e_{N}$ for every $x \in \partial \Omega$ and that there is a constant $M_{\psi}$ such that

$$
\sup _{h \geq 1}\left\{\left\|D^{2} \psi_{h}\right\|_{2, \infty},\left\|D^{2} \psi_{h}^{-1}\right\|_{2, \infty}\right\} \leq M_{\psi}
$$

Notice also that we may assume that for all $h \geq 1$ the inclusion $U_{h} \subset \subset V_{h}$ holds, and that we can choose a $C^{2}$ domain $E$ such that $\psi_{h}\left(U_{h} \cap \Omega\right) \subset E \subset B_{1}^{-}(0)$.

Using the transformation $\hat{f}(y):=f\left(\psi_{h}^{-1}(y)\right)$ for a generic $f$ defined in $\Omega \cap V_{h}$, and since $v_{h}$ is the solution of (3.8), we get that for every $h \geq 1$ the function $\hat{v}_{h}(t, y)=$ $\eta_{h}\left(\psi_{h}^{-1}(y)\right) u\left(t, \psi_{h}^{-1}(y)\right)$ is the solution of the following initial-boundary value problem with homogeneous Neumann boundary conditions

$$
\begin{cases}\partial_{t} w-\hat{\mathcal{A}} w=\hat{\mathcal{A}}_{h} \hat{v} & \text { in }(0,+\infty) \times E, \\ w(0)=\hat{\eta}_{h} \hat{u}_{\sigma} & \text { in } E, \\ \frac{\partial w}{\partial v}=0 & \text { in }(0,+\infty) \times \partial E,\end{cases}
$$

where $\hat{\mathcal{A}}$ is the operator defined on $B_{1}(0)$ as follows

$$
\hat{\mathcal{A}} w:=\operatorname{div}(\hat{A} D w)+\langle\hat{B}, D w\rangle+\hat{C} w
$$


defined by the coefficients (here for $\hat{\mathcal{A}}$ and its coefficients we omit the index $h$ to simplify the notations and by analogy with (3.8))

$$
\begin{aligned}
\hat{A}(y):= & \left(D \psi_{h}\right)\left(\psi_{h}^{-1}(y)\right) \cdot A\left(\psi_{h}^{-1}(y)\right) \cdot\left(D \psi_{h}\right)^{t}\left(\psi_{h}^{-1}(y)\right), \\
(\hat{B}(y))_{l}:= & \operatorname{Tr}\left[\left(D \psi_{h}\right)\left(\psi_{h}^{-1}(y)\right) \cdot A\left(\psi_{h}^{-1}(y)\right) \cdot H^{l}\left(\psi_{h}^{-1}(y)\right) \cdot\left(D \psi_{h}^{-1}\right)^{t}(y)\right] \\
& +\operatorname{Tr}\left[\left(D \psi_{h}\right)\left(\psi_{h}^{-1}(y)\right) \cdot G^{j}(y)\right]\left(D \psi_{h}\right)_{j l}^{t}\left(\psi_{h}^{-1}(y)\right)-\frac{\partial}{\partial y_{j}}\left[\hat{A}_{j l}(y)\right] \\
& +\left[\left(D \psi_{h}\right)\left(\psi_{h}^{-1}(y)\right) \cdot B\left(\psi_{h}^{-1}(y)\right)\right]_{l}, \\
\hat{C}(y):= & C\left(\psi_{h}^{-1}(y)\right),
\end{aligned}
$$

where $H_{k i}^{l}=D_{k i}^{2}\left(\psi_{h}\right)_{l}$ and $G_{k i}^{j}=D_{k} A_{i j}\left(\psi_{h}^{-1}(y)\right)$ and (see (3.9))

$\hat{\mathcal{A}}_{h} \hat{u}(t)=-2\left\langle A\left(\psi_{h}^{-1}(y)\right)\left(D \psi_{h}\right)^{t} D \hat{\eta}_{h},\left(D \psi_{h}\right)^{t} D \hat{u}(t)\right\rangle-\hat{u}(t)\left[\operatorname{div}\left(\hat{A} D \hat{\eta}_{h}\right)+\left\langle\hat{B}, D \hat{\eta}_{h}\right\rangle\right]$.

Now, as done before for $h=0$, differentiating the equation (now $D_{k}=\frac{\partial}{\partial y_{k}}$ ) we obtain that $D_{k} \hat{v}_{h}$ solves $\partial_{t}\left(D_{k} \hat{v}_{h}(t)\right)-\hat{\mathcal{A}}\left(D_{k} \hat{v}_{h}(t)\right)=\hat{\mathcal{A}}_{h}^{k} \hat{u}(t)$, where $\hat{\mathcal{A}}_{h}^{k} \hat{v}$ can be obtained by taking the corresponding term in (3.10). Associated with this operator, we can consider the problem

$$
\begin{cases}\partial_{t} w-\hat{\mathcal{A}} w=\hat{\mathcal{A}}_{h}^{k} \hat{u}(t) & \text { in }(0, \infty) \times E, \\ w(0)=D_{k}\left(\hat{\eta}_{h} \hat{u}_{\sigma}\right) & \text { in } E, \\ \frac{\partial w}{\partial v}=0 & \text { in }(0, \infty) \times \partial E .\end{cases}
$$

The function $D_{k} \hat{v}_{h}$ satisfies the equation and the initial condition. Notice that if $k \neq N$ also the boundary condition is satisfied since $\hat{v}_{h}=0$ in a neighbourhood of $\partial E \cap\left\{y \in \mathbb{R}^{N} \mid y_{N}<0\right\}$, in the other part of $\partial E$ the operator $D_{k}$ is a tangential derivative and $\frac{\partial \hat{v}_{h}}{\partial y_{N}}$ is constant for $y_{N}=0$. Denote by $S$ the semigroup which gives the solution of this problem and notice that the estimates (3.2) hold for $S(t)$, see Remark 3.2. Then

$$
D_{k} \hat{v}_{h}(t)=S(t) D_{k} \hat{v}_{h}(0)+\int_{0}^{t} S(t-s) \hat{\mathcal{A}}_{h}^{k} \hat{u}(s) d s .
$$

Differentiating (3.15) with respect to $D_{j}$ for any $j$, we have then proved that the following holds

$$
D_{k j}^{2} \hat{v}_{h}(t)=D_{j} S(t) D_{k} \hat{v}_{h}(0)+\int_{0}^{t} D_{j} S(t-s) \hat{\mathcal{A}}_{h}^{k} \hat{u}(s) d s .
$$

Thus, as for $v_{0}(t)$, we have for $(k, j) \neq(N, N)$

$$
\begin{aligned}
\left\|D_{k j}^{2} \hat{v}_{h}(t)\right\|_{L^{1}(E)} & \leq \frac{c_{2}}{\sqrt{t}}\left\|D_{k}\left(\hat{\eta}_{h} \hat{u}_{\sigma}\right)\right\|_{L^{1}(E)}+\int_{0}^{t} \frac{c_{2}}{\sqrt{t-s}}\left\|\hat{\mathcal{A}}_{h}^{k} \hat{u}(s)\right\|_{L^{1}(E)} d s . \\
& \leq \frac{c}{\sqrt{t \sigma}}\|\hat{u}\|_{L^{1}(E)}+\int_{0}^{t} \frac{c_{2}}{\sqrt{t-s}}\left\|\hat{\mathcal{A}}_{h}^{k} \hat{u}(s)\right\|_{L^{1}(E)} d s .
\end{aligned}
$$


We now estimate $D_{N N}^{2} \hat{v}_{h}(t)$. Since

$$
\begin{aligned}
\hat{A}_{N N} D_{N N}^{2} \hat{v}_{h}(t)= & \hat{\mathcal{A}} \hat{v}_{h}(t)-\sum_{(i, j) \neq(N, N)} \hat{A}_{i j} D_{i j}^{2} \hat{v}_{h}(t)-\sum_{i, j=1}^{N}\left(D_{i} \hat{A}_{i j}\right) D_{j} \hat{v}_{h}(t) \\
& -\sum_{i=1}^{N} \hat{B}_{i} D_{i} \hat{v}_{h}(t)-\hat{C} \hat{v}_{h}(t)
\end{aligned}
$$

and since $\hat{A}$ is uniformly elliptic with ellipticity constant proportional to $\lambda$, we can find a constant $c$ (depending only on $N, M_{1}, \lambda, \partial \Omega$ ) such that

$$
\begin{aligned}
\left\|D_{N N}^{2} \hat{v}_{h}(t)\right\|_{L^{1}(E)}= & \| \frac{1}{\hat{A}_{N N}}\left(\hat{\mathcal{A}} \hat{v}_{h}(t)-\sum_{(i, j) \neq(N, N)} \hat{A}_{i j} D_{i j}^{2} \hat{v}_{h}(t)\right. \\
& \left.-\sum_{i, j=1}^{N}\left(D_{i} \hat{A}_{i j}\right) D_{j} \hat{v}_{h}(t)-\sum_{i=1}^{N} \hat{B}_{i} D_{i} \hat{v}_{h}(t)-\hat{C} \hat{v}_{h}(t)\right) \|_{L^{1}(E)} \\
\leq c & {\left[\sum_{(i, j) \neq(N, N)}\left\|D_{i j}^{2} \hat{v}_{h}(t)\right\|_{L^{1}(E)}+\left\|\hat{\mathcal{A}} \hat{v}_{h}(t)\right\|_{L^{1}(E)}+\left\|D \hat{v}_{h}(t)\right\|_{L^{1}(E)}+\left\|\hat{v}_{h}(t)\right\|_{L^{1}(E)}\right] . }
\end{aligned}
$$

Summing up, we may argue in the same way as for $h=0$, and get

$$
\begin{aligned}
& \left\|D^{2} \hat{v}_{h}(t)\right\|_{L^{1}(E)} \\
& \leq c^{\prime}\left[\frac{1}{\sqrt{t}}\left\|u_{\sigma} \circ \psi_{h}^{-1}\right\|_{W^{1,1}(E)}+\int_{0}^{t} \frac{1}{\sqrt{t-s}}\left\|D^{2} \hat{u}(s)\right\|_{L^{1}(E)} d s+\left\|\hat{\mathcal{A}} \hat{v}_{h}(t)\right\|_{L^{1}(E)}\right] \\
& \leq c^{\prime}\left[\frac{1}{\sqrt{t \sigma}}\left\|u \circ \psi_{h}^{-1}\right\|_{L^{1}(E)}+\int_{0}^{t} \frac{1}{\sqrt{t-s}}\left\|D^{2} \hat{u}(s)\right\|_{L^{1}(E)} d s+\left\|\hat{\mathcal{A}} \hat{v}_{h}(t)\right\|_{L^{1}(E)}\right],
\end{aligned}
$$

where $c^{\prime}=c\left(M, M_{1}, M_{\psi}, N, c_{2}, c_{v}\right)$. Coming back to $\Omega \cap U_{h}$ we obtain

$$
\begin{aligned}
& \left\|D^{2} v_{h}(t)\right\|_{L^{1}\left(\Omega \cap U_{h}\right)} \\
& \leq c^{\prime \prime}\left[\frac{1}{\sqrt{t}}\left\|u_{\sigma}\right\|_{W^{1,1}\left(\Omega \cap U_{h}\right)}+\int_{0}^{t} \frac{1}{\sqrt{t-s}}\left\|D^{2} u(s)\right\|_{L^{1}\left(\Omega \cap U_{h}\right)} d s+\left\|\mathcal{A} v_{h}(t)\right\|_{L^{1}\left(\Omega \cap U_{h}\right)}\right] \\
& \leq c^{\prime \prime}\left[\frac{1}{\sqrt{t \sigma}}\|u\|_{L^{1}\left(\Omega \cap U_{h}\right)}+\int_{0}^{t} \frac{1}{\sqrt{t-s}}\left\|D^{2} u(s)\right\|_{L^{1}\left(\Omega \cap U_{h}\right)} d s+\left\|\mathcal{A} v_{h}(t)\right\|_{L^{1}\left(\Omega \cap U_{h}\right)}\right],
\end{aligned}
$$


where $c^{\prime \prime}$ depends on $M, M_{1}, M_{\psi}, N, c_{2}, c_{v}$. Now, using (3.2) and (3.6), we have

$$
\begin{aligned}
& \left\|D^{2} u(t)\right\|_{L^{1}(\Omega)}=\left\|D^{2}\left(\sum_{h=0}^{\infty} v_{h}(t)\right)\right\|_{L^{1}(\Omega)}=\left\|\sum_{h=0}^{\infty} D^{2} v_{h}(t)\right\|_{L^{1}(\Omega)} \\
& \leq \kappa c^{\prime \prime}\left[\frac{1}{\sqrt{t}}\left\|u_{\sigma}\right\|_{W^{1,1}(\Omega)}+\int_{0}^{t} \frac{1}{\sqrt{t-s}}\left\|D^{2} u(s)\right\|_{L^{1}(\Omega)} d s+\|\mathcal{A} u(t)\|_{L^{1}(\Omega)}\right] \\
& \leq c^{\prime \prime \prime}\left[\frac{1}{\sqrt{t \sigma}}\|u\|_{L^{1}(\Omega)}+\int_{0}^{t} \frac{1}{\sqrt{t-s}}\left\|D^{2} u(s)\right\|_{L^{1}(\Omega)} d s+\frac{1}{\sqrt{t \sigma}}\|u\|_{L^{1}(\Omega)}\right],
\end{aligned}
$$

where $c^{\prime \prime \prime}$ depends on $\kappa, c^{\prime \prime}, c_{3}$.

Using Gronwall's generalized inequality (see for instance [12, Lemma 7.1.1]), and taking $\sigma=t$ we get $\left\|D^{2} u(t)\right\|_{L^{1}(\Omega)} \leq c_{5} t^{-1}\|u\|_{L^{1}(\Omega)}$ for every $t \in(0,1)$.

We can use the previous proposition to characterize some interpolation spaces.

Proposition 3.4 For each $\alpha \in(0,1)$, we have that

$$
D_{\mathcal{A}}(\alpha, 1)=\left(L^{1}(\Omega), W^{2,1}(\Omega) \cap W_{A, v}^{1,1}(\Omega)\right)_{\alpha, 1},
$$

where $W_{A, v}^{1,1}$ is the closure of $\left\{u \in C^{1}(\bar{\Omega}):\langle A \nabla u, v\rangle=0\right.$ on $\left.\partial \Omega\right\}$ with respect to the topology of $W^{1,1}$ and $v$ denotes the outward unit normal to $\partial \Omega$. Moreover, if $\alpha \in(0,1 / 2)$, there holds

$$
\left(L^{1}(\Omega), W^{2,1}(\Omega) \cap W_{A, v}^{1,1}(\Omega)\right)_{\alpha, 1}=W^{2 \alpha, 1}(\Omega),
$$

where $W^{2 \alpha, 1}(\Omega)$ is the fractional Sobolev space.

Proof The first part of the proposition can be proved as in [10, Theorem 3.1]. Next, for short, we define for an open and regular set $\omega \subset \mathbf{R}^{N}$ the space

$$
X_{\alpha}(\omega)=\left(L^{1}(\omega), W^{2,1}(\omega) \cap W_{A, v}^{1,1}(\omega)\right)_{\alpha, 1}
$$

endowed with the norm

$$
\|u\|_{X_{\alpha}}:=\int_{0}^{+\infty} \frac{K(t, u)}{t^{1+\alpha}} d t, K(t, u): \inf _{\substack{a+b=u \\ a \in L^{1}(\omega) \\ b \in W^{2,1}(\omega) \cap W_{A, v}^{1,1}(\omega)}}\left(\|u\|_{L^{1}(\omega)}+t\|u\|_{W^{2,1}(\omega)}\right) .
$$

The first step is to prove that the equality $\left(L^{1}(\Omega), D(\mathcal{A})\right)_{\alpha, 1}=X_{\alpha}(\Omega)$ holds for every $\alpha \in(0,1)$, and this can be done following again the proof of Theorem 3.1 in [10] and using Proposition 3.3.

The second step is to prove that $X_{\alpha}(\Omega)=W^{2 \alpha, 1}(\Omega)$ for $\alpha \in(0,1 / 2)$. For the result in the case when $\omega=\mathbf{R}^{N}$ we refer to [5, Theorem 4.3.6]. Then it is easy to prove the same result for $\omega=\mathbf{R}_{+}^{N}=\left\{x \in \mathbf{R}^{N} \mid x_{N}>0\right\}$ by extending a function $u: \mathbf{R}_{+}^{N} \rightarrow \mathbf{R}$ to a function $\tilde{u}: \mathbf{R}^{N} \rightarrow \mathbf{R}$ as $\tilde{u}\left(x_{1}, \ldots x_{N-1}, x_{N}\right)=u\left(x_{1}, \ldots x_{N-1},-x_{N}\right)$ for $x_{N}<0$.

Now we consider the same partition of unity $\left\{\eta_{h}\right\}_{h}$ associated with the covering $\left\{U_{h}\right\}_{h}$ of $\Omega$ considered in the proof of Proposition 3.3. Then, for a given function $u$ defined in $\Omega$ and writing $u$ as $\sum_{h=0}^{+\infty} u \eta_{h}$, we can prove that $u \eta_{0} \in X_{\alpha}(\Omega)$ if and only if $u \eta_{0} \in W^{2 \alpha, 1}(\Omega)$. For every $h \geq 1$ we can find $\psi_{h}: B_{+}(0) \rightarrow U_{h} \cap \Omega$, where $B_{+}(0)=B(0,1) \cap \mathbf{R}_{+}^{N}$, and 
prove that $v_{h}:=u \eta_{h} \circ \psi_{h}$ belongs to $X_{\alpha}\left(\mathbf{R}_{+}^{N}\right)$ if and only if belongs to $W^{2 \alpha, 1}\left(\mathbf{R}_{+}^{N}\right)$, by which $u \eta_{h} \in X_{\alpha}(\Omega)$ if and only if $u \eta_{h} \in W^{2 \alpha, 1}(\Omega)$. Now to conclude we have to show that $u \in X_{\alpha}(\Omega)$ if and only if $u \in W^{2 \alpha, 1}(\Omega)$. Notice that the result is immediate if $\Omega$ is bounded, since in that case the covering $\left\{U_{h}\right\}_{h}$ is finite.

Suppose first that $u \in X_{\alpha}(\Omega)$. Since $X_{\alpha}(\Omega)$ continuously embeds in $L^{1}(\Omega)$, it is sufficient to estimate the seminorm $|u|_{\alpha, \Omega}$. Since $u \in X_{\alpha}(\Omega)$ we also have that $u \eta_{h} \in X_{\alpha}(\Omega)$ for each $h \in \mathbf{N}$. Indeed, $u \in W^{2 \alpha, 1}(\Omega)$ if and only if $u \in L^{1}(\Omega)$ and for every $\rho>0$ one has

$$
\int_{|x-y|<\rho} \frac{|u(x)-u(y)|}{|x-y|^{N+2 \alpha}} d x d y<+\infty
$$

and in particular

$$
|u|_{\alpha, \Omega} \leq \frac{2}{\rho^{N+2 \alpha}}\|u\|_{L^{1}(\Omega)}+\int_{|x-y|<\rho} \frac{|u(x)-u(y)|}{|x-y|^{N+2 \alpha}} d x d y .
$$

Fix $\rho>0$ and let $\left\{B_{m}\right\}_{m}$ be a countable covering of $\Omega$ such that $\left\{B_{m} \times B_{m}\right\}_{m}$ is a covering of $\{(x, y) \in \Omega \times \Omega:|x-y|<\rho\}$ with bounded overlapping. Notice that, since $\left\{U_{h}\right\}_{h}$ has a bounded overlapping, the equality

$$
u(x)-u(y)=\sum_{h} u(x) \eta_{h}(x)-\sum_{k} u(y) \eta_{k}(y)
$$

for $x, y \in B_{m}$ becomes

$$
u(x)-u(y)=\sum_{h \in H_{m}}\left(u(x) \eta_{h}(x)-u(y) \eta_{h}(y)\right),
$$

where $H_{m}$ is a finite set of indices, whose cardinality is a constant $C$ which depends only on the bound of the overlapping. Moreover we can choose the covering $\left\{B_{m}\right\}_{m}$ in such a way that there is a constant $\kappa$, depending only on the overlapping of the $B_{m}$, with

$$
\begin{aligned}
& \sum_{m}\|w\|_{L^{1}\left(B_{m}\right)} \leq \kappa\|w\|_{L^{1}(\Omega)} \quad \text { for every } w \in L^{1}(\Omega), \\
& \sum_{m}\|w\|_{W^{2,1}\left(B_{m}\right)} \leq \kappa\|w\|_{W^{2,1}(\Omega)} \quad \text { for every } w \in W^{2,1}(\Omega), \\
& \sum_{h \in H_{m}}\left\|u \eta_{h}\right\|_{L^{1}(\Omega)} \leq \kappa\|u\|_{L^{1}\left(B_{m}\right)}, \\
& \sum_{h \in H_{m}}\left\|u \eta_{h}\right\|_{W^{2,1}\left(B_{m}\right)} \leq \kappa\left\|\eta_{h}\right\|_{W^{2, \infty}}\|u\|_{W^{2,1}\left(B_{m}\right)} .
\end{aligned}
$$

Then

$$
\begin{aligned}
\int_{|x-y|<\rho} \frac{|u(x)-u(y)|}{|x-y|^{N+2 \alpha}} d x d y & \leq \sum_{m=1}^{\infty} \int_{B_{m}} d x \int_{B_{m}} \frac{\left|\sum_{h \in H_{m}}\left(u(x) \eta_{h}(x)-u(y) \eta_{h}(y)\right)\right|}{|x-y|^{N+2 \alpha}} d y \\
& \leq \sum_{m=1}^{\infty} \sum_{h \in H_{m}} \int_{B_{m}} d x \int_{B_{m}} \frac{\left|u(x) \eta_{h}(x)-u(y) \eta_{h}(y)\right|}{|x-y|^{N+2 \alpha}} d y
\end{aligned}
$$

Since the functions $v_{h}:=u \eta_{h} \circ \psi_{h}$ belong both to $\left(L^{1}\left(\mathbf{R}_{+}^{N}\right), W^{2,1}\left(\mathbf{R}_{+}^{N}\right) \cap W_{A, \nu}^{1,1}\left(\mathbf{R}_{+}^{N}\right)\right)_{\alpha, 1}$ and $W^{2 \alpha, 1}\left(\mathbf{R}_{+}^{N}\right)$, and in $\mathbf{R}_{+}^{N}$ the norms of $W^{2 \alpha, 1}\left(\mathbf{R}_{+}^{N}\right)$ and $X_{\alpha}\left(\mathbf{R}_{+}^{N}\right)$ are equivalent, we get a constant $\kappa_{0}$, depending only on the norm of the embedding of $X_{\alpha}\left(\mathbf{R}_{+}^{N}\right)$ in $W^{2 \alpha, 1}\left(\mathbf{R}_{+}^{N}\right)$ and 
$\psi_{h}$, such that

$$
\int_{B_{m}} d x \int_{B_{m}} \frac{\left|u(x) \eta_{h}(x)-u(y) \eta_{h}(y)\right|}{|x-y|^{N+2 \alpha}} d y \leq \kappa_{0} \int_{0}^{+\infty} \frac{1}{t^{1+\alpha}} K\left(t, u \eta_{h}\right) d t,
$$

where $K$ is defined in (3.19). By definition of $K(t, \cdot)$ and by (3.22) we get

$$
\begin{aligned}
& \sum_{h \in H_{m}} K\left(t, u \eta_{h}\right)=\sum_{h \in H_{m}} \inf _{\substack{\tilde{a}+\tilde{b}=u \eta_{h} \\
\tilde{a} \in L^{1}(\Omega), \tilde{b} \in W^{2,1}(\Omega)}}\left(\|\tilde{a}\|_{L^{1}(\Omega)}+t\|\tilde{b}\|_{W^{2,1}(\Omega)}\right) \\
& \leq \sum_{h \in H_{m}} \inf _{\substack{a+b=u \\
a \in L^{1}(\Omega), b \in W^{2,1}(\Omega)}}\left(\left\|a \eta_{h}\right\|_{L^{1}(\Omega)}+t\left\|b \eta_{h}\right\|_{W^{2,1}(\Omega)}\right) \\
& \leq \inf _{\substack{a+b=u \\
a \in L^{1}(\Omega), b \in W^{2,1}(\Omega)}} \sum_{h \in H_{m}}\left(\left\|a \eta_{h}\right\|_{L^{1}(\Omega)}+t\left\|b \eta_{h}\right\|_{W^{2,1}(\Omega)}\right) \\
& \leq \kappa_{1} \inf _{\substack{a+b=u \\
a \in L^{1}(\Omega), b \in W^{2,1}(\Omega)}}\left(\|a\|_{L^{1}\left(B_{m}\right)}+t\|b\|_{W^{2,1}\left(B_{m}\right)}\right),
\end{aligned}
$$

where $\kappa_{1}$ depends on $\kappa$ and $\left\|\eta_{h}\right\|_{W^{2, \infty}(\Omega)}$. Summing up on $m$ we get, by (3.21),

$$
\sum_{m=1}^{+\infty} \sum_{h \in H_{m}} K\left(t, u \eta_{h}\right) \leq \kappa \kappa_{1} K(t, u) .
$$

Then by (3.23), (3.24) and using the last estimate we get

$$
\begin{aligned}
\int_{|x-y|<\rho} \frac{|u(x)-u(y)|}{|x-y|^{N+2 \alpha}} d x d y & \leq \sum_{m=1}^{+\infty} \sum_{h \in H_{m}} \kappa_{0} \int_{0}^{+\infty} \frac{1}{t^{1+\alpha}} K\left(t, u \eta_{h}\right) d t \\
& \leq \kappa \kappa_{0} \kappa_{1} \int_{0}^{+\infty} \frac{1}{t^{1+\alpha}} K(t, u) d t=\kappa \kappa_{0} \kappa_{1}\|u\|_{X_{\alpha}(\Omega)},
\end{aligned}
$$

whence $X_{\alpha}(\Omega) \subset W^{2 \alpha, 1}(\Omega)$. To prove the reverse inclusion, consider $\left\{\eta_{h}, U_{h}\right\}_{h}$ as before. First of all observe that, as done in (3.20), we can estimate for each $\rho>0$

$$
\left|u \eta_{h}\right|_{\alpha, \Omega} \leq \frac{2}{\rho^{N+2 \alpha}}\|u\|_{L^{1}\left(U_{h}\right)}+\int_{|x-y|<\rho} \frac{\left|u(x) \eta_{h}(x)-u(y) \eta_{h}(y)\right|}{|x-y|^{N+2 \alpha}} d x d y .
$$

Adding and subtracting $u(x) \eta_{h}(y)$ we can estimate

$$
\begin{aligned}
& \int_{|x-y|<\rho} \frac{\left|u(x) \eta_{h}(x)-u(y) \eta_{h}(y)\right|}{|x-y|^{N+2 \alpha}} d x d y \\
& \leq \int_{\Omega \times \Omega}\left[\operatorname{Lip}\left(\eta_{h}\right) \frac{|u(x)|}{|x-y|^{N-1+2 \alpha}} \chi_{A_{h, \rho}}(x, y)+\frac{|u(x)-u(y)|}{|x-y|^{N+2 \alpha}} \chi_{\Omega \times U_{h}}(x, y)\right] d x d y,
\end{aligned}
$$

where $A_{h, \rho}=\left(U_{h} \times \Omega \cup \Omega \times U_{h}\right) \cap\{(x, y) \in \Omega \times \Omega:|x-y|<\rho\}$. Then, choosing $\rho$ small enough in order that the $\rho$-enlarged sets $U_{h}^{\rho}$ have the same overlapping as the $U_{h}$ 's 
and $A_{h, \rho} \subset U_{h}^{\rho} \times U_{h}^{\rho}$, we get

$$
\left\|u \eta_{h}\right\|_{W^{2 \alpha, 1}(\Omega)} \leq \kappa_{2}\|u\|_{L^{1}\left(U_{h}^{\rho}\right)}+\int_{U_{h}} d y \int_{B(y, \rho)} \frac{|u(x)-u(y)|}{|x-y|^{N+2 \alpha}} d x,
$$

where $\kappa_{2}$ depends (only) on $\left\|\eta_{h}\right\|_{W^{1, \infty}}, \alpha, \rho, N$. Since the overlapping is bounded we can find two constants $\kappa_{3}, \kappa_{4}$ such that

$$
\sum_{h}\left\|u \eta_{h}\right\|_{W^{2 \alpha, 1}(\Omega)} \leq \kappa_{3}\left[\|u\|_{L^{1}(\Omega)}+\int_{\Omega} d y \int_{B(y, \rho)} \frac{|u(x)-u(y)|}{|x-y|^{N+2 \alpha}} d x\right] \leq \kappa_{4}\|u\|_{W^{2 \alpha, 1}(\Omega)} .
$$

Then for each $\epsilon>0$ we can find $\tilde{a}_{h} \in L^{1}(\Omega), \tilde{b}_{h} \in W^{2,1}(\Omega)$ such that $\tilde{a}_{h}+\tilde{b}_{h}=u \eta_{h}$ and $\left\|\tilde{a}_{h}\right\|_{L^{1}(\Omega)}+t\left\|\tilde{b}_{h}\right\|_{W^{2,1}(\Omega)} \leq K\left(t, u \eta_{h}\right)+\epsilon 2^{-h}$. Define $a=\sum_{h} \tilde{a}_{h}$ and $b=\sum_{h} \tilde{b}_{h}$. Then $a+b=u$ and

$$
K(t, u) \leq\|a\|_{L^{1}(\Omega)}+t\|b\|_{W^{2,1}(\Omega)} \leq \sum_{h}\left\|\tilde{a}_{h}\right\|_{L^{1}(\Omega)}+t\left\|\tilde{b}_{h}\right\|_{W^{2,1}(\Omega)} \leq \sum_{h} K\left(t, u \eta_{h}\right)+\epsilon
$$

and then $K(t, u) \leq \sum_{h} K\left(t, u \eta_{h}\right)$. Now, as before, since the functions $v_{h}$ are in $W^{2 \alpha, 1}\left(\mathbf{R}_{+}^{N}\right)$ and in $\mathbf{R}_{+}^{N}$, the norms of $W^{2 \alpha, 1}\left(\mathbf{R}_{+}^{N}\right)$ and $X_{\alpha}\left(\mathbf{R}_{+}^{N}\right)$ are equivalent, there exists a constant $\kappa_{5}$, depending only on the norm of the embedding of $W^{2 \alpha, 1}\left(\mathbf{R}_{+}^{N}\right)$ in $X_{\alpha}\left(\mathbf{R}_{+}^{N}\right)$ and $\psi_{h}$, such that

$$
\int_{0}^{+\infty} \frac{1}{t^{1+\alpha}} K\left(t, u \eta_{h}\right) d t \leq \kappa_{5} \int_{\Omega} d x \int_{\Omega} \frac{\left|u(x) \eta_{h}(x)-u(y) \eta_{h}(y)\right|}{|x-y|^{N+2 \alpha}} d y .
$$

Therefore there is a constant $\kappa_{6}$ (depending only on $\kappa_{4}$ and $\kappa_{5}$ )

$$
\begin{aligned}
\int_{0}^{+\infty} \frac{1}{t^{1+\alpha}} K(t, u) d t & \leq \int_{0}^{+\infty} \frac{1}{t^{1+\alpha}} \sum_{h=1}^{+\infty} K\left(t, u \eta_{h}\right) d t \\
& \leq \kappa_{5} \sum_{h=1}^{+\infty}\left\|u \eta_{h}\right\|_{W^{2 \alpha, 1}(\Omega)} \leq \kappa_{6}\|u\|_{W^{2 \alpha, 1}(\Omega)} .
\end{aligned}
$$

Using Proposition 3.4, we can improve estimate of Proposition 3.3, under additional assumption on the initial datum; in fact, we have the following.

Proposition 3.5 Let $\Omega, \mathcal{A}, \mathcal{B}$ be as in Sect. 2. Assume, in addition, $A \in W^{2, \infty}(\Omega)$ and $B, C \in W^{1, \infty}(\Omega)$; then, there exist $\delta \in(1 / 2,1)$ and $c_{6}$ depending on $N, \lambda, \Omega,\|A\|_{2, \infty}$, $\|B\|_{1, \infty},\|C\|_{1, \infty}, c_{1}, c_{2}, c_{3} c_{v}$ such that for every $t \in(0,1)$ and $u \in W^{1,1}(\Omega)$ we have

$$
t^{\delta}\left\|D^{2} T(t) u\right\|_{L^{1}(\Omega)} \leq c_{6}\|u\|_{W^{1,1}(\Omega)} .
$$

Proof We can repeat the proof of Proposition 3.3 until the first inequality in (3.18), with $\sigma=0$, so that we have

$$
\left\|D^{2} u(t)\right\|_{L^{1}(\Omega)} \leq \kappa c^{\prime \prime}\left[\frac{1}{\sqrt{t}}\|u\|_{W^{1,1}(\Omega)}+\int_{0}^{t} \frac{1}{\sqrt{t-s}}\left\|D^{2} u(s)\right\|_{L^{1}(\Omega)} d s+\|\mathcal{A} u(t)\|_{L^{1}(\Omega)}\right] .
$$


Using [14, Proposition 2.2.9], we get that for any $\alpha, \beta \in(0,1)$ there is $c$ such that $t^{1-\alpha+\beta} \| \mathcal{A} T$

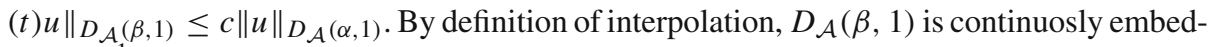
ded in $L^{1}(\Omega)$ for any $\beta \in(0,1)$. Using the fact that $D_{\mathcal{A}}(\alpha, 1)$ is the fractional Sobolev space $W^{2 \alpha, 1}(\Omega)$ for $\alpha<1 / 2$ and that $W^{1,1}(\Omega)$ embeds in $W^{2 \alpha, 1}(\Omega)$ for such $\alpha$, we obtain, with constants $c$ that may change from a line to the other,

$$
\begin{aligned}
\|\mathcal{A} T(t) u\|_{L^{1}(\Omega)} & \leq c\|\mathcal{A} T(t) u\|_{D_{\mathcal{A}}(\beta, 1)} \leq \frac{c}{t^{1-\alpha+\beta}}\|u\|_{D_{\mathcal{A}}(\alpha, 1)} \\
& =\frac{c}{t^{1-\alpha+\beta}}\|u\|_{W^{2 \alpha, 1}(\Omega)} \leq \frac{c}{t^{1-\alpha+\beta}}\|u\|_{W^{1,1}(\Omega)} .
\end{aligned}
$$

We choose then $\alpha \in(0,1 / 2)$ and $\beta \in(0,1)$ is such a way that $\delta=1-\alpha+\beta \in(1 / 2,1)$, and (3.27) becomes

$$
\left\|D^{2} u(t)\right\|_{L^{1}(\Omega)} \leq \frac{c}{t^{\delta}}\|u\|_{L^{1}(\Omega)}+\int_{0}^{t} \frac{c}{\sqrt{t-s}}\left\|D^{2} u(s)\right\|_{L^{1}(\Omega)} d s .
$$

Again, (3.26) follows from Gronwall's lemma.

\section{$4 B V$ functions}

Let us recall the definition and the basic properties of functions with possibly weighted bounded variation on $\Omega$. For $u \in L^{1}(\Omega)$, given a symmetric positive definite matrix $P=$ $\left(P_{i j}\right)_{i, j=1}^{N}$, we can define the weighted total variation, following [3], by setting

$$
|D u|_{P}(\Omega)=\sup \left\{\int_{\Omega} u \operatorname{div} \psi d x: \psi \in C_{c}^{1}\left(\Omega, \mathbb{R}^{N}\right),\left\|P^{-1 / 2} \psi\right\|_{\infty} \leq 1\right\}
$$

and say that $u$ has finite total weighted variation, $u \in B V_{P}(\Omega)$, if $|D u|_{P}(\Omega)<+\infty$. A set $E$ is said to have finite weighted perimeter if $\left|D \chi_{E}\right|_{P}(\Omega)<+\infty$. In this case, its total variation measure is the perimeter of $E$ and it is denoted also by $P_{P}(E, \Omega)=\left|D \chi_{E}\right|_{P}(\Omega)$. Notice that if $P$ has entries $P_{i j} \in C^{1}(\Omega)$, then the total variation can be equivalently defined by

$$
|D u|_{P}(\Omega)=\sup \left\{\int_{\Omega} u \operatorname{div}\left(P^{1 / 2} \phi\right) d x: \phi \in C_{c}^{1}\left(\Omega, \mathbb{R}^{N}\right),\|\phi\|_{\infty} \leq 1\right\} .
$$

Of course, if $P$ is the identity matrix then $|D u|_{P}$ reduces to (1.3) and the weighted perimeter reduces to the classical perimeter, and in this case we write $u \in B V(\Omega)$ and drop the $P$ everywhere. The space $B V_{P}(\Omega)$ turns out to be a Banach space with norm

$$
\|u\|_{B V_{P}}=\|u\|_{L^{1}(\Omega)}+|D u|_{P}(\Omega) .
$$

The norm topology is in some respects too strong, since for instance smooth functions are not dense with respect to it. Nevertheless, a classical weaker approximation result is given by the Anzellotti-Giaquinta theorem, see, e.g., [2, Theorem 3.9]. It states that for every $u \in B V(\Omega)$ there exists a sequence of functions $\left(u_{n}\right)_{n} \subset C^{\infty}(\Omega)$ such that

$$
\left\|u-u_{n}\right\|_{L^{1}(\Omega)} \rightarrow 0, \quad \int_{\Omega}\left|D u_{n}\right| d x \rightarrow|D u|(\Omega) ;
$$

such a sequence is said to converge in variation to $u$. 
Let us recall a particular case of [7, Lemma 2.4], i.e., the following coarea formula:

$$
|D u|_{P}(\Omega)=\int_{\mathbb{R}} P_{P}(\{u>\tau\}, \Omega) d \tau,
$$

which we use later.

Henceforth, we assume the following:

(H4) $P$ is a symmetric strictly positive definite matrix with $P_{i j} \in C_{b}(\bar{\Omega})$.

Under the above hypothesis, the seminorms $|D u|(\Omega)$ and $|D u|_{P}(\Omega)$ are equivalent.

We also notice that if $u$ is regular, then the equality

$$
|D u|_{P}(\Omega)=\int_{\Omega}|D u(x)|_{P} d x,
$$

holds, where $|D u(x)|_{P}$ is defined in (2.1). We notice that the weighted total variation is the supremum of the $L^{1}(\Omega)$ continuous functionals

$$
u \mapsto \int_{\Omega} u \operatorname{div} \psi d x
$$

and therefore it is $L^{1}(\Omega)$ lower semicontinuous, i.e.,

$$
|D u|_{P}(\Omega) \leq \liminf _{n \rightarrow+\infty}\left|D u_{n}\right|_{P}(\Omega)
$$

for any sequence $\left(u_{n}\right)_{n}$ with $u_{n} \rightarrow u$ in $L^{1}(\Omega)$. In particular, if $(T(t))_{t \geq 0}$ is a strongly continuous semigroup on $L^{1}(\Omega)$, then

$$
\left|D u_{0}\right|_{P}(\Omega) \leq \liminf _{t \rightarrow 0} \int_{\Omega}\left|D T(t) u_{0}\right|_{P} d x .
$$

The Anzellotti-Giaquinta theorem can be adapted also to the case of weighted $B V$ functions, as is done in the following result.

Proposition 4.1 Let $\Omega, P=\left(P_{i j}\right)_{i, j=1}^{N}$ be as above, and let $Q=\left(Q_{i j}\right)_{i, j=1}^{N}$ be an elliptic matrix with $Q_{i j} \in C_{b}^{1}(\bar{\Omega})$. Then, for every $u \in B V_{P}(\Omega)$ there exists a sequence of functions $\left(v_{n}\right)_{n} \subset C_{Q}(\Omega)$ such that

$$
\lim _{n \rightarrow \infty}\left\|u-v_{n}\right\|_{L^{1}(\Omega)}=0, \quad \lim _{n \rightarrow \infty} \int_{\Omega}\left|D v_{n}\right|_{P} d x=|D u|_{P}(\Omega) .
$$

Proof The proof goes as the classical one, except that we have to modify the usual approximation sequence in a neighbourhood of the boundary of $\Omega$. The assumption on the regularity on $\partial \Omega$ is used to modify the approximating sequence to make it constant in the direction $Q v$.

Remark 4.2 A particular case of Proposition 4.1 is given when $Q=A$; in this case we have that $C_{A}(\Omega) \subset D(\mathcal{A})$ (it is a core), and then the weighted $B V$ functions can be approximated in variation via functions in the domain of the operator $\mathcal{A}$.

For the weighted total variation also the following continuity property under uniform convergence holds. 
Proposition 4.3 Let $P=\left(P_{i j}\right)_{i, j=1}^{N}$ be a symmetric $\lambda$-elliptic matrix valued function and let $\left(P_{(n)}\right)_{n \in \mathbb{N}}$ be a sequence of matrices valued functions uniformly convergent to $P$. Then, for every $u \in L^{1}(\Omega)$ the following holds:

$$
\lim _{n \rightarrow+\infty}|D u|_{P_{(n)}}(\Omega)=|D u|_{P}(\Omega) .
$$

Proof We denote by $c_{n}=\left\|P^{-1 / 2}-P_{(n)}^{-1 / 2}\right\|_{\infty}$; by the uniform convergence, we have that $c_{n} \rightarrow 0$ as $n \rightarrow+\infty$; moreover, we may assume that the $P_{(n)}$ are $(\lambda+1 / n)$-elliptic, that is $(\lambda+1 / n)^{-1}|\xi|^{2} \leq\left|P_{(n)}^{1 / 2} \xi\right|^{2} \leq(\lambda+1 / n)|\xi|^{2}$, or, simply defining $w=P_{(n)}^{1 / 2} \xi,(\lambda+$ $1 / n)^{-1 / 2}|w| \leq\left|P_{(n)}^{-1 / 2} w\right| \leq \sqrt{\lambda+1 / n}|w|$. Then, if $\psi \in C_{c}^{1}\left(\Omega, \mathbb{R}^{N}\right)$ with $\left\|P_{(n)}^{-1 / 2} \psi\right\|_{\infty} \leq 1$, we get

$$
\begin{aligned}
\left\|P^{-1 / 2} \psi\right\|_{\infty} & \leq\left\|P_{(n)}^{-1 / 2} \psi\right\|_{\infty}+\left\|\left(P^{-1 / 2}-P_{(n)}^{-1 / 2}\right) \psi\right\|_{\infty} \leq\left\|P_{(n)}^{-1 / 2} \psi\right\|_{\infty}+c_{n}\|\psi\|_{\infty} \\
& \leq\left\|P_{(n)}^{-1 / 2} \psi\right\|_{\infty}+c_{n} \sqrt{\lambda+1 / n}\left\|P_{(n)}^{-1 / 2} \psi\right\|_{\infty} \leq 1+c_{n} \sqrt{\lambda+1 / n} .
\end{aligned}
$$

By definition of weighted variation, we get

$$
\int_{\Omega} u \operatorname{div} \psi d x \leq\left(1+c_{n} \sqrt{\lambda+1 / n}\right)|D u|_{P}(\Omega),
$$

whence $|D u|_{P_{(n)}}(\Omega) \leq\left(1+c_{n} \sqrt{\lambda+1 / n}\right)|D u|_{P}(\Omega)$. With a similar computation, we also get $|D u|_{P}(\Omega) \leq\left(1+c_{n} \sqrt{\lambda}\right)|D u|_{P_{(n)}}(\Omega)$, and then (4.4) follows by letting $n \rightarrow+\infty$.

\section{The first characterization of $B V$ functions}

In this section we prove that for $u_{0} \in L^{1}(\Omega)$ the equality

$$
\lim _{t \rightarrow 0} \int_{\Omega}\left|D T(t) u_{0}\right|_{P} d x=\left|D u_{0}\right|_{P}(\Omega)
$$

holds, where $(T(t))_{t \geq 0}$ is the semigroup generated by the operator $(\mathcal{A}, D(\mathcal{A}))$ in $(2.4)$ and $|D u|_{P}(\Omega)$ is defined in (4.1).

Notice that, by the result obtained in Sect. 4, equality (5.1) holds for $u_{0} \in D(\mathcal{A})$, see (3.3), and moreover by semicontinuity inequality (4.3) always holds.

In this section we require more regularity on the coefficients $A_{i j}$, and replace hypothesis (H3) with the following:

(H3) ${ }^{\prime} \quad A_{i j} \in W^{2, \infty}(\Omega), B, C \in L^{\infty}(\Omega)$.

We need the following result, which gives a localized version of (5.1). Since all the results of this section are stated with $t \in(0,1)$, we may use the results in Sect. 3 without assuming $C \leq 0$, as explained at the beginning of that section.

Proposition 5.1 Let $v \in D(\mathcal{A})$, where $\mathcal{A}$ is as in (2.4), with coefficients $A_{i j} \in W^{2, \infty}(\Omega)$, $B_{i}, C \in W^{1, \infty}(\Omega)$. Let $P=\left(P_{i j}\right)_{i, j=1}^{N}$ be a non-negative $\lambda$-elliptic matrix with $P_{i j} \in$ $W^{1, \infty}(\Omega)$ and $P_{i j}=A_{i j}$ on $\partial \Omega$. Then for every $\eta \in C_{b}^{1}(\bar{\Omega})$, $\eta$ non-negative, there exists $a$ constant

$$
c_{7}=c\left(M_{1}, N,\|P\|_{1, \infty},\|\eta\|_{1, \infty}, \lambda\right)
$$


such that

$$
\int_{\Omega} \eta|D T(t) v|_{P} d x \leq \int_{\Omega} \eta|D v|_{P} d x+c_{7} t^{1-\delta}\|v\|_{W^{1,1}(\Omega)}
$$

holds for every $t \in(0,1)$, where $\delta \in(1 / 2,1)$ is the parameter in (3.26).

Proof For $v \in D(\mathcal{A})$ and $\eta \in C_{b}^{1}(\bar{\Omega}), \eta \geq 0$, we define the function $F_{\eta}:(0,1) \rightarrow \mathbb{R}$ by

$$
F_{\eta}(t)=\int_{\Omega} \eta|D T(t) v|_{P} d x .
$$

This function is differentiable since $T(t) v$ is regular for every $t>0$ and the equality

$$
\partial_{t}|D T(t) v|_{P}=\frac{1}{|D T(t) v|_{P}}\langle P D T(t) v, D \mathcal{A} T(t) v\rangle
$$

holds for a.e. $x \in \Omega$. Moreover, $T(t) v \in D(\mathcal{A})$ for every $t>0$ and then

$$
\mathcal{A} T(t) v=T\left(\frac{t}{2}\right) \mathcal{A} T\left(\frac{t}{2}\right) v ;
$$

this implies also that $\mathcal{A} T(t) v \in D(\mathcal{A})$. Then, thanks to (3.3) and from the fact that

$$
\frac{|\langle P D T(t) v, D \mathcal{A} T(t) v\rangle|}{|D T(t) v|_{P}} \leq|D \mathcal{A} T(t) v|_{P},
$$

we can differentiate under the integral sign. Denoting by $u(t, x)$ the solution $(T(t) v)(x)$, we obtain

$$
\begin{aligned}
F_{\eta}^{\prime}(t)= & \frac{d}{d t} \int_{\Omega} \eta|D u|_{P} d x=\int_{\Omega} \frac{\eta}{|D u|_{P}}\langle P D u, D \mathcal{A} u\rangle d x \\
= & \sum_{i, j, h, k=1}^{N} \int_{\Omega} \eta \frac{P_{i j} D_{j} u D_{i}\left(D_{h}\left(A_{h k} D_{k} u\right)\right)}{|D u|_{P}} d x \\
& +\sum_{i, j, h=1}^{N} \int_{\Omega} \eta \frac{P_{i j} D_{j} u D_{i}\left(B_{h} D_{h} u\right)}{|D u|_{P}} d x+\sum_{i, j=1}^{N} \int_{\Omega} \eta \frac{P_{i j} D_{j} u D_{i}(C u)}{|D u|_{P}} d x \\
\left(I_{1}\right)= & \sum_{i, j, h, k=1}^{N} \int_{\Omega} \eta \frac{P_{i j} D_{j} u\left(D_{i h}^{2} A_{h k} D_{k} u+D_{h} A_{h k} D_{i k}^{2} u+D_{i} A_{h k} D_{h k}^{2} u\right)}{|D u|_{P}} d x \\
& +\sum_{i, j, h, k=1}^{N} \int_{\Omega} \eta \frac{1}{|D u|_{P}} P_{i j} D_{j} u A_{h k} D_{i h k}^{3} u d x \\
& +\sum_{i, j, h, k=1}^{N} \int_{\Omega} \eta \frac{1}{|D u|_{P}} P_{i j} D_{j} u\left(D_{i} B_{h} D_{h} u+B_{h} D_{i h}^{2} u\right) d x \\
& +\sum_{i, j, h, k=1}^{N} \int_{\Omega} \eta \frac{1}{|D u|_{P}} P_{i j} D_{j} u\left(D_{i} C u+C D_{i} u\right) d x .
\end{aligned}
$$


Notice that there is a constant $c_{8}=c\left(N, M_{1},\|\eta\|_{\infty},\|P\|_{\infty}\right)$ such that

$$
\left|I_{1}\right|+\left|I_{3}\right|+\left|I_{4}\right| \leq c_{8}\|u\|_{W^{2,1}(\Omega)} .
$$

It remains to estimate $I_{2}$; integrating by parts with respect to $x_{k}$, we have that

$$
\begin{gathered}
\sum_{i, j, h, k=1}^{N} \int_{\Omega} \frac{\eta}{|D u|_{P}} P_{i j} D_{j} u A_{h k} D_{i h k}^{3} u d x \\
\left(I I_{1}\right)=\frac{1}{2} \sum_{i, j, h, k, l, m=1}^{N} \int_{\Omega} \frac{\eta}{|D u|_{P}^{3}} P_{i j} D_{j} u A_{h k} D_{i h}^{2} u D_{k} P_{l m} D_{m} u D_{l} u d x \\
\left(I I_{2}\right) \quad+\sum_{i, j, h, k, l, m=1}^{N} \int_{\Omega} \frac{\eta}{|D u|_{P}^{3}} P_{i j} D_{j} u A_{h k} D_{i h}^{2} u P_{l m} D_{m} u D_{k l}^{2} u d x \\
\left(I I_{3}\right) \quad-\sum_{i, j, h, k=1}^{N} \int_{\Omega} \frac{\eta}{|D u|_{P}}\left(D_{k} P_{i j} D_{j} u A_{h k}+P_{i j} D_{j} u D_{k} A_{h k}\right) D_{i h}^{2} u d x \\
\left(I I_{4}\right) \quad-\sum_{i, j, h, k=1}^{N} \int_{\Omega} \frac{\eta}{|D u|_{P}} P_{i j} D_{k j}^{2} u A_{h k} D_{i h}^{2} u d x \\
\left(I I_{5}\right) \quad-\sum_{i, j, h, k=1}^{N} \int_{\Omega} \frac{1}{|D u|_{P}} P_{i j} D_{j} u A_{h k} D_{i h}^{2} u D_{k} \eta d x \\
\left(I I_{6}\right) \quad+\sum_{i, j, h, k=1}^{N} \int_{\partial \Omega} \frac{\eta}{|D u|_{P}} P_{i j} D_{j} u A_{h k} D_{i h}^{2} u v_{k} d \mathcal{H}^{N-1} .
\end{gathered}
$$

This implies the existence of a constant $c_{9}=c\left(M_{1},\|P\|_{1, \infty},\|\eta\|_{1, \infty}\right)$, such that

$$
\left|I I_{1}\right|+\left|I I_{3}\right|+\left|I I_{5}\right| \leq c_{9} \int_{\Omega}\left|D^{2} u\right| d x .
$$

Notice that for $I I_{2}$ we have

$$
\begin{aligned}
\sum_{i, j, k, l, m=1}^{N} P_{i j} D_{j} u A_{h k} D_{i h}^{2} u P_{l m} D_{m} u D_{k l}^{2} u & =\left\langle D^{2} u A D^{2} u P D u, P D u\right\rangle \\
& =\left\langle P^{1 / 2} D^{2} u A D^{2} u P^{1 / 2}\left(P^{1 / 2} D u\right), P^{1 / 2} D u\right\rangle
\end{aligned}
$$

and for $\mathrm{II}_{4}$ we can write

$$
\begin{aligned}
\sum_{i, j, h, k=1}^{N} P_{i j} D_{k j}^{2} u A_{h k} D_{i h}^{2} u & =\sum_{i, j, h, k=1}^{N} P_{i m}^{1 / 2} P_{m j}^{1 / 2} D_{k j}^{2} u A_{h k} D_{i h}^{2} u \\
& =\operatorname{Tr}\left(P^{1 / 2} D^{2} u A D^{2} u P^{1 / 2}\right),
\end{aligned}
$$


where $\operatorname{Tr}$ denotes the trace of a matrix. Then

$$
\begin{aligned}
I I_{2}+I I_{4}= & \int_{\Omega} \frac{1}{|D u|_{P}}\left(\left\langle P^{1 / 2} D^{2} u A D^{2} u P^{1 / 2} \frac{P^{1 / 2} D u}{|D u|_{P}}, \frac{P^{1 / 2} D u}{|D u|_{P}}\right\rangle\right. \\
& \left.-\operatorname{Tr}\left(P^{1 / 2} D^{2} u A D^{2} u P^{1 / 2}\right)\right) \eta d x \leq 0
\end{aligned}
$$

since $P^{1 / 2} D^{2} u A D^{2} u P^{1 / 2}$ is positive definite because

$$
\left\langle\left(P^{1 / 2} D^{2} u A D^{2} u P^{1 / 2}\right) \xi, \xi\right\rangle=\left\langle A^{1 / 2} D^{2} u P^{1 / 2} \xi, A^{1 / 2} D^{2} u P^{1 / 2} \xi\right\rangle .
$$

Finally, for the term $I I_{6}$, we notice that

$$
\begin{aligned}
& \sum_{i, j, h, k=1}^{N} P_{i j} D_{j} u A_{h k} D_{i h}^{2} u v_{k}=\sum_{i=1}^{N}\left(\sum_{h, k=1}^{N} A_{h k} D_{i h}^{2} u v_{k} \sum_{j=1}^{N} P_{i j} D_{j} u\right) \\
& =\sum_{i=1}^{N} \sum_{h, k=1}^{N}\left(D_{i}\left(A_{h k} D_{h} u v_{k}\right)-D_{h} u D_{i}\left(A_{h k} v_{k}\right)\right) \sum_{j=1}^{N} P_{i j} D_{j} u \\
& =\langle D\langle A D u, v\rangle, P D u\rangle-\langle D(A v) D u, P D u\rangle=-\langle D(A v) D u, P D u\rangle
\end{aligned}
$$

since $P \equiv A$ on $\partial \Omega$. Observe that the regularity of the boundary and the ellipticity of $A_{i j}$ imply that there exists a constant $c_{10}=c\left(L, M_{1}\right)$ such that $|D(A \nu)| \leq c_{10}$. As a consequence, we obtain that

$$
\begin{aligned}
& \left|\sum_{i, j, h, k=1}^{N} \int_{\partial \Omega} \frac{1}{|D u|_{P}} \eta P_{i j} D_{j} u A_{h k} D_{i h}^{2} u v_{k} d \mathcal{H}^{N-1}\right| \\
& =\left|\int_{\partial \Omega} \frac{1}{|D u|_{P}} \eta\langle D(A v) D u, P D u\rangle d \mathcal{H}^{N-1}\right| \leq c_{10} \int_{\partial \Omega} \eta|D u|_{P} d \mathcal{H}^{N-1} \\
& \leq c_{10}\|\eta\|_{\infty} \sqrt{\lambda} \int_{\partial \Omega}|D u| d \mathcal{H}^{N-1} \leq c_{11} \int_{\Omega}\left[|D u|+\left|D^{2} u\right|\right] d x,
\end{aligned}
$$

where $c_{11}=c\left(M_{1}, L, \lambda,\|\eta\|_{\infty}, c_{\Omega}\right)$, where $c_{\Omega}$ is introduced in (2.3).

Taking now into account that $u(t, x)$ satisfies (3.2) and (3.26), we have proved there is a constant $c$ such that for every $t \in(0,1)$ the inequality

$$
F_{\eta}^{\prime}(t)=\frac{d}{d t} \int_{\Omega} \eta|D u|_{P} d x \leq c t^{-\delta}\left\|u_{0}\right\|_{W^{1,1}(\Omega)}
$$

holds. Then, by integration (5.2) follows.

Theorem 5.2 Assume (H1), (H2), (H3)', (H4), and let $(T(t))_{t \geq 0}$ be the semigroup generated by $(\mathcal{A}, D(\mathcal{A}))$ in $L^{1}(\Omega)$. Then, for every $u_{0} \in L^{1}(\Omega)$, the equality

$$
\lim _{t \rightarrow 0} \int_{\Omega}\left|D T(t) u_{0}(x)\right|_{P} d x=\left|D u_{0}\right|_{P}(\Omega)
$$

holds. In particular, $u_{0}$ belongs to $B V(\Omega)$ if and only if the above limit is finite. 
Proof We start first by assuming that $P_{i j} \in C_{b}^{2}(\bar{\Omega})$ and considering the operator $\hat{\mathcal{A}} u=$ $\operatorname{div}(A D u)$, i.e., $B_{i}=C=0, i=1, \ldots N$, and the generated semigroup, denoted $\hat{T}$. Thanks to (4.3), we have only to prove that

$$
\limsup _{t \rightarrow 0} \int_{\Omega}\left|D \hat{T}(t) u_{0}(x)\right|_{P} d x \leq\left|D u_{0}\right|_{P}(\Omega),
$$

which is trivially satified if $u_{0} \in L^{1}(\Omega) \backslash B V(\Omega)$. We then consider $u_{0} \in B V(\Omega)$. Fix $\varepsilon>0$ and consider two open neighbourhoods $U \subset V$ of $\partial \Omega$ with disjoint boundaries such that, if we take $S^{\prime}=\Omega \cap U$ and $S=\Omega \cap \bar{V}$, we get

$$
\left|D u_{0}\right|_{P}(S)<\varepsilon .
$$

Let then $\eta \in C^{2}(\Omega)$ be a function such that

$$
0 \leq \eta \leq 1, \quad \eta \equiv 1 \text { on } S^{\prime}, \quad \eta \equiv 0 \text { on } \Omega \backslash S
$$

and define the matrix

$$
P_{A}=\eta^{2} A+\left(1-\eta^{2}\right) P .
$$

By Proposition 4.1 there exists a sequence

$$
\begin{aligned}
\left(u_{n}\right)_{n} & \subset\left\{v \in C^{\infty}(\Omega) \cap C^{1}(\bar{\Omega}):\langle A D v, v\rangle=0 \text { on } \partial \Omega\right\} \\
& =\left\{v \in C^{\infty}(\Omega) \cap C^{1}(\bar{\Omega}):\left\langle P_{A} D v, v\right\rangle=0 \text { on } \partial \Omega\right\} \subset D(\mathcal{A})
\end{aligned}
$$

such that $u_{n} \rightarrow u_{0}$ in $L^{1}(\Omega)$ and

$$
\lim _{n \rightarrow+\infty} \int_{\Omega}\left|D u_{n}\right|_{P} d x=\left|D u_{0}\right|_{P}(\Omega) .
$$

Notice that since $P$ is $\lambda$-elliptic we get

$$
\int_{\Omega}\left|D u_{n}\right| d x \leq \sqrt{\lambda} \int_{\Omega}\left|D u_{n}\right|_{P} d x
$$

and then there exists $M>0$ such that

$$
\left\|u_{n}\right\|_{W^{1,1}(\Omega)} \leq M .
$$

Since $\Omega \backslash S$ is an open set, by lower semicontinuity we have

$$
\left|D u_{0}\right|_{P}(\Omega \backslash S) \leq \liminf _{n \rightarrow+\infty} \int_{\Omega \backslash S}\left|D u_{n}\right|_{P} d x
$$

and also

$$
\int_{S}\left|D u_{n}\right|_{P} d x=\int_{\Omega}\left|D u_{n}\right|_{P} d x-\int_{\Omega \backslash S}\left|D u_{n}\right|_{P} d x,
$$

whence

$$
\begin{aligned}
\limsup _{n \rightarrow+\infty} \int_{S}\left|D u_{n}\right|_{P} d x & \leq \lim _{n \rightarrow+\infty} \int_{\Omega}\left|D u_{n}\right|_{P} d x-\liminf _{n \rightarrow+\infty} \int_{\Omega \backslash S}\left|D u_{n}\right|_{P} d x \\
& \leq\left|D u_{0}\right|_{P}(\Omega)-\left|D u_{0}\right|_{P}(\Omega \backslash S)=\left|D u_{0}\right|_{P}(S) .
\end{aligned}
$$


This proves that

$$
\limsup _{n \rightarrow+\infty} \int_{S}\left|D u_{n}\right|_{P} d x \leq\left|D u_{0}\right|_{P}(S)
$$

by the $\lambda$-ellipticity of $A$ and $P$, we get that $|\xi|_{A} \leq \sqrt{\lambda}|\xi|_{P}$ therefore the following holds:

$$
\limsup _{n \rightarrow+\infty} \int_{S}\left|D u_{n}\right|_{A} d x=\limsup _{n \rightarrow+\infty} \int_{S}\left\langle A D u_{n}, D u_{n}\right\rangle^{1 / 2} d x \leq \lambda \limsup _{n \rightarrow+\infty} \int_{S}\left|D u_{n}\right|_{P} d x
$$

whence by (5.8) and (5.6)

$$
\limsup _{n \rightarrow+\infty} \int_{S}\left|D u_{n}\right|_{A} d x \leq \lambda \varepsilon
$$

We also notice that

$$
\begin{aligned}
|\xi|_{P}^{2} & =\langle P \xi, \xi\rangle=\left\langle P_{A} \xi, \xi\right\rangle+\left\langle\left(P-P_{A}\right) \xi, \xi\right\rangle \\
& =\left\langle P_{A} \xi, \xi\right\rangle+\eta^{2}\langle(P-A) \xi, \xi\rangle=|\xi|_{P_{A}}^{2}+\eta^{2}\langle(P-A) \xi, \xi\rangle
\end{aligned}
$$

and, since $P$ and $A$ are $\lambda$-elliptic,

$$
|\langle(P-A) \xi, \xi\rangle| \leq 2 \lambda|\xi|^{2} \leq 2 \lambda^{2}|\xi|_{A}^{2}, \quad \forall \xi \in \mathbb{R}^{N} .
$$

We have then obtained that $|\xi|_{P} \leq|\xi|_{P_{A}}+\lambda \sqrt{2} \eta|\xi|_{A}$ and as a consequence

$$
\int_{\Omega}\left|D \hat{T}(t) u_{n}\right|_{P} d x \leq \int_{\Omega}\left|D \hat{T}(t) u_{n}\right|_{P_{A}} d x+\lambda \sqrt{2} \int_{\Omega} \eta\left|D \hat{T}(t) u_{n}\right|_{A} d x .
$$

We can apply Proposition 5.1 to both terms in the right hand side in order to obtain, using (5.7), that

$$
\int_{\Omega}\left|D \hat{T}(t) u_{n}\right|_{P} d x \leq \int_{\Omega}\left|D u_{n}\right|_{P_{A}} d x+\lambda \sqrt{2} \int_{\Omega} \eta\left|D u_{n}\right|_{A} d x+(1+\lambda \sqrt{2}) c_{7} M t^{1-\delta} .
$$

By definition of $P_{A}$, we have that

$$
|\xi|_{P_{A}}^{2}=\eta^{2}|\xi|_{A}^{2}+\left(1-\eta^{2}\right)|\xi|_{P}^{2}, \quad \forall \xi \in \mathbb{R}^{N}
$$

and then

$$
\int_{\Omega}\left|D u_{n}\right|_{P_{A}} d x \leq \int_{\Omega} \eta\left|D u_{n}\right|_{A} d x+\int_{\Omega} \sqrt{1-\eta^{2}}\left|D u_{n}\right|_{P} d x \leq \int_{S}\left|D u_{n}\right|_{A} d x+\int_{\Omega}\left|D u_{n}\right|_{P} d x .
$$

We have then obtained the following estimate

$$
\int_{\Omega}\left|D \hat{T}(t) u_{n}\right|_{P} d x \leq \int_{\Omega}\left|D u_{n}\right|_{P} d x+(1+\lambda \sqrt{2}) \int_{S}\left|D u_{n}\right|_{A} d x+(1+\lambda \sqrt{2}) c_{7} M t^{1-\delta} \text {. }
$$

Using (5.9), (5.10) and the fact that $\hat{T}(t) u_{n} \rightarrow \hat{T}(t) u_{0}$ in $L^{1}(\Omega)$ as $n \rightarrow+\infty$, we get

$$
\begin{aligned}
\int_{\Omega}\left|D \hat{T}(t) u_{0}\right|_{P} d x & \leq \liminf _{n \rightarrow+\infty} \int_{\Omega}\left|D \hat{T}(t) u_{n}\right|_{P} d x \leq \limsup _{n \rightarrow+\infty} \int_{\Omega}\left|D \hat{T}(t) u_{n}\right|_{P} d x \\
& \leq\left|D u_{0}\right|_{P}(\Omega)+\lambda(1+\lambda \sqrt{2}) \varepsilon+(1+\lambda \sqrt{2}) c_{7} M t^{1-\delta}
\end{aligned}
$$


and the result for $P$ regular then follows by letting $t \rightarrow 0$, since $\varepsilon$ is arbitrary. The case with $P_{i j} \in C_{b}(\bar{\Omega})$ is a consequence of the approximation result given in Proposition 4.3.

Finally, we consider non zero coefficients $B_{i}$ and $C$ and $\mathcal{A} u=\operatorname{div}(A D u)+\langle B, D u\rangle+C u$ with $B_{i}, C \in L^{\infty}(\Omega), i=1, \ldots N$. Notice that the boundary operators associated with $\mathcal{A}$ and $\hat{\mathcal{A}}$ as in (2.5) coincide, and then the set $C_{A}(\Omega)$ defined in (2.2) is a core both for $(\mathcal{A}, D(\mathcal{A}))$ and $(\hat{\mathcal{A}}, D(\hat{\mathcal{A}}))$. We denote by $(T(t))_{t \geq 0}$ the semigroup generated by $(\mathcal{A}, D(\mathcal{A}))$. Notice that if we define $\hat{u}(t):=\hat{T}(t) u_{0}$ and $u=T(t) u_{0}$, with $u_{0} \in C_{A}(\Omega)$, the function $w:=\hat{u}-u$ is the solution of the problem

$$
\begin{cases}\partial_{t} w-\mathcal{A} w=\mathcal{E} \hat{u}:=-\langle B, D \hat{u}\rangle-C \hat{u} & \text { in }(0, \infty) \times \Omega, \\ w(0)=0 & \text { in } \Omega, \\ \langle A D w, v\rangle=0 & \text { in }(0, \infty) \times \partial \Omega .\end{cases}
$$

Thus, since $w(t)=\int_{0}^{t} T(t-s) \mathcal{E} \hat{u}(s) d s$, we get

$$
D w(t)=D(\hat{u}-u)(t)=\int_{0}^{t} D T(t-s) \mathcal{E} \hat{u}(s) d s
$$

and then using (3.2)

$$
\begin{aligned}
\left\|D \hat{T}(t) u_{0}-D T(t) u_{0}\right\|_{L^{1}(\Omega)} & \leq c_{2}\left\|\mathcal{E} \hat{T}(t) u_{0}\right\|_{L^{1}(\Omega)} \int_{0}^{t} \frac{1}{\sqrt{t-s}} d s \\
& \leq 2 c_{2} \sqrt{t}\left(\|B\|_{\infty}\left\|D \hat{T}(t) u_{0}\right\|_{L^{1}(\Omega)}+\|C\|_{\infty}\left\|\hat{T}(t) u_{0}\right\|_{L^{1}(\Omega)}\right) .
\end{aligned}
$$

Since $\left\|\hat{T}(t) u_{0}\right\|_{L^{1}(\Omega)} \rightarrow\left\|u_{0}\right\|_{L^{1}(\Omega)}$ and $\lim \sup _{t \rightarrow 0}\left\|D \hat{T}(t) u_{0}\right\|_{L^{1}(\Omega)}$ is bounded we can conclude that $\lim _{t \rightarrow 0}\left\|D \hat{T}(t) u_{0}-D T(t) u_{0}\right\|_{L^{1}(\Omega)}=0$ and consequently, for $v \in C_{A}(\Omega)$, it follows

$$
\begin{gathered}
\limsup _{t \rightarrow 0} \int_{\Omega}|D T(t) v|_{P} d x \leq \limsup _{t \rightarrow 0} \int_{\Omega}|D \hat{T}(t) v|_{P} d x \\
+\lim _{t \rightarrow 0} \int_{\Omega}|D \hat{T}(t) v-D T(t) v|_{P} d x=\int_{\Omega}|D v|_{P} d x .
\end{gathered}
$$

The thesis then follows from the density of $C_{A}(\Omega)$ in $B V_{P}(\Omega)$ (see Proposition 4.1); given $u_{0} \in B V_{P}(\Omega)$, we take a sequence $\left(u_{n}\right) \subset C_{A}(\Omega)$ approximating $u_{0}$ in $P$-variation. Then, using (5.11) with $u_{n}$ in place of $u_{0}$ and (5.10), we get

$$
\begin{aligned}
& \int_{\Omega}\left|D T(t) u_{n}\right|_{P} d x \leq \int_{\Omega}\left|D \hat{T}(t) u_{n}\right|_{P} d x+\int_{\Omega}\left|D T(t) u_{n}-D \hat{T}(t) u_{n}\right|_{P} d x \\
& \quad \leq\left(1+2 c_{2} \sqrt{t}\|B\|_{\infty}\right) \int_{\Omega}\left|D u_{n}\right|_{P} d x
\end{aligned}
$$




$$
\begin{aligned}
& +(1+\lambda \sqrt{2})\left(1+2 c_{2} \sqrt{t}\|B\|_{\infty}\right) \int_{S}\left|D u_{n}\right|_{A} d x \\
& +(1+\lambda \sqrt{2})\left(1+2 c_{2} \sqrt{t}\|B\|_{\infty}\right) c_{7} M t^{1-\delta}+2 c_{2} \sqrt{\lambda t}\|C\|_{\infty} \int_{\Omega}\left|\hat{T}(t) u_{n}\right| d x
\end{aligned}
$$

and consequently it follows

$$
\begin{aligned}
\left|D u_{0}\right|_{P}(\Omega) \leq & \liminf _{t \rightarrow 0} \int_{\Omega}\left|D T(t) u_{0}\right|_{P} d x \leq \limsup _{t \rightarrow 0} \limsup _{n \rightarrow+\infty} \int_{\Omega}\left|D T(t) u_{n}\right|_{P} d x \\
\leq & \limsup _{t \rightarrow 0}\left\{\left(1+2 c_{2} \sqrt{t}\|B\|_{\infty}\right)\left|D u_{0}\right|_{P}(\Omega)+(1+\lambda \sqrt{2})\left(1+2 c_{2} \sqrt{t}\|B\|_{\infty}\right) \varepsilon\right. \\
& \left.+(1+\lambda \sqrt{2})\left(1+2 c_{2} \sqrt{t}\|B\|_{\infty}\right) c_{7} M t^{1-\delta}+c_{2} \sqrt{\lambda t}\|C\|_{\infty}\left\|u_{0}\right\|_{L^{1}(\Omega)}\right\} \\
= & \left|D u_{0}\right|_{P}(\Omega)+(1+\lambda \sqrt{2}) \varepsilon .
\end{aligned}
$$

The result then follows since $\varepsilon$ is arbitrary.

We end with the discussion of the simplest application of Theorem 5.2.

Example 5.1 Of course, the simplest case is with $A=P=I, B=C=0$, i.e., $(T(t))_{t \geq 0}$ is the heat semigroup generated by the Neumann Laplacian and the total variation is the classical (non-weighted) one. In this case, it is easily seen that $F(t)=\left\|D T(t) u_{0}\right\|_{L^{1}(\Omega)}$ is decreasing (as is the case if $\Omega=\mathbb{R}^{N}$ ), provided that $\Omega$ is convex. In fact, our computations significantly simplify and go as follows, where as in the proof of Theorem 5.2 we set $u(t, x)=$ $\left(T(t) u_{0}\right)(x)$ :

$$
\begin{aligned}
F^{\prime}(t) & =\int_{\Omega} \partial_{t}|D u| d x=\int_{\Omega} \frac{1}{|D u|}\left\langle D u, D \partial_{t} u\right\rangle d x=\int_{\Omega} \frac{1}{|D u|} \sum_{i, k} D_{i} u D_{i} D_{k k}^{2} u d x \\
& =\int_{\partial \Omega} \frac{1}{|D u|} \sum_{i, k} D_{i} u D_{i k}^{2} u v_{k} d \mathcal{H}^{N-1}-\int_{\Omega} \sum_{i, k} D_{k} \frac{D_{i} u}{|D u|} D_{i k}^{2} u d x \\
& =-\int_{\partial \Omega} \frac{1}{|D u|}\langle D v D u, D u\rangle d \mathcal{H}^{N-1}-\int_{\Omega}\left[\left|D^{2} u \frac{D u}{|D u|}\right|^{2}-\operatorname{Tr}\left(D^{2} u\right)^{2}\right] d x \leq 0,
\end{aligned}
$$

where we have taken into account (5.3), (5.4) and the fact that if $\Omega$ is convex then all the curvatures (i.e., the eigenvalues of the matrix $D v$ ) are non-negative.

Notice that it has been proved in [11, Theorem 2.16] that there is a (non-convex) $\Omega$ such that $F^{\prime}(0)>0$.

\section{A second characterization of $B V$ functions}

In this section we give a characterization of $B V$ functions using in a different way the semigroup generated by the operator $\mathcal{A}$, in the spirit of [15]; more precisely, we prove that for every $u \in L^{1}(\Omega)$ equality (1.5) holds. The right hand side there significantly simplifies if $u=\chi_{E}$ is the characteristic function of a measurable set $E \subset \mathbb{R}^{N}$ of finite perimeter, and 
reads

$$
\lim _{t \rightarrow 0} \sqrt{\frac{\pi}{t}} \int_{E^{c} \cap \Omega} T(t) \chi_{E} d x=\int_{\mathcal{F} E \cap \Omega}\left|A^{1 / 2}(x) v_{E}(x)\right| d \mathcal{H}^{N-1}(x),
$$

where $\mathcal{F} E$ is the reduced boundary of $E$. Indeed, we first prove (6.1), and then, using it in connection with the coarea formula, we deduce (1.5). Let us recall some of the main notions on sets of finite perimeter; for a detailed description see for instance [2]. We denote by $\mathcal{F} E$ the reduced boundary of $E$, defined as the set

$$
\mathcal{F} E=\left\{x \in \operatorname{supp}\left|D \chi_{E}\right|: \exists \lim _{\varrho \rightarrow 0} \frac{D \chi_{E}\left(B_{\varrho}(x)\right)}{\left|D \chi_{E}\right|\left(B_{\varrho}(x)\right)}=v_{E}(x) \text {, and }\left|v_{E}(x)\right|=1\right\} .
$$

Moreover, we denote by $E^{\alpha}$ the set of points of $\mathbb{R}^{N}$ with density $\alpha$ at $x$, that is

$$
E^{\alpha}=\left\{x \in \mathbb{R}^{N}: \exists \lim _{\varrho \rightarrow 0} \frac{\left|E \cap B_{\varrho}(x)\right|}{\left|B_{\varrho}(x)\right|}=\alpha\right\} ;
$$

the essential boundary is then defined as $\partial^{*} E=\mathbb{R}^{N} \backslash\left(E^{0} \cup E^{1}\right)$. The main properties of sets of finite perimeter we need are that $\mathcal{F} E \subset E^{1 / 2}$ and that $\mathcal{H}^{N-1}\left(\partial^{*} E \backslash \mathcal{F} E\right)=0$.

For every $t>0$ and $x_{0} \in \Omega$, we set

$$
\Omega^{t, x_{0}}=\frac{\Omega-x_{0}}{\sqrt{t}}=\left\{y \in \mathbb{R}^{N}: x_{0}+\sqrt{t} y \in \Omega\right\}
$$

and, given $f: \Omega \rightarrow \mathbb{R}, f^{t, x_{0}}(y)=f\left(x_{0}+\sqrt{t} y\right)$ with this notation, we define the operator $\mathcal{A}^{t, x_{0}}$ on $\Omega^{t, x_{0}}$ by

$$
\begin{aligned}
\mathcal{A}^{t, x_{0}}(y) v(y)= & \operatorname{div}\left(A^{t, x_{0}}(y) D v(y)\right)+\sqrt{t}\left\langle B^{t, x_{0}}(y), D v(y)\right\rangle+t C^{t, x_{0}}(y) v(y) \\
= & \sum_{h, k=1}^{N} A_{h k}\left(x_{0}+\sqrt{t} y\right) \frac{\partial^{2} v}{\partial y^{h} \partial y^{k}}(y)+\sqrt{t} \sum_{k=1}^{N}\left(\sum_{h=1}^{N} D_{h} A_{h k}\left(x_{0}+\sqrt{t} y\right)\right) \frac{\partial v}{\partial y^{k}}(y) \\
& +\sqrt{t} \sum_{h=1}^{N} B_{h}\left(x_{0}+\sqrt{t} y\right) \frac{\partial v}{\partial y^{h}}(y)+t C\left(x_{0}+\sqrt{t} y\right) v(y)
\end{aligned}
$$

and the operator $\mathcal{A}^{x}$ on $\mathbb{R}^{N}$ by

$$
\mathcal{A}^{x} v(y)=\sum_{h, k=1}^{N} A_{h k}(x) \frac{\partial^{2} v}{\partial y^{h} \partial y^{k}}(y) .
$$

By setting $x=x_{0}+\sqrt{t} y$, it is easily seen that $\mathcal{A}^{t, x_{0}}(y)=t \mathcal{A}(x)$. We have the following Lemma.

Lemma 6.1 Setting $u(s, x)=T(s) u_{0}(x)$, we can define the function $v:(0,+\infty) \times \Omega^{t, x_{0}} \rightarrow$ $\mathbb{R}$ by $v(s, y)=u\left(t s, x_{0}+\sqrt{t} y\right)$; then $v$ is the solution of the problem

$$
\begin{cases}\partial_{s} w=\mathcal{A}^{t, x_{0}}(y) w & \text { in }(0,+\infty) \times \Omega^{t, x_{0}}, \\ w(0, y)=u_{0}^{t, x_{0}}(y) & \text { in } \Omega^{t, x_{0}} \\ \left\langle A^{t, x_{0}} D w, v\right\rangle=0 & \text { in }(0,+\infty) \times \partial \Omega^{t, x_{0}} .\end{cases}
$$


Proof By definition, we have $v(0, y)=u\left(0, x_{0}+\sqrt{t} y\right)=u_{0}\left(x_{0}+\sqrt{t} y\right)=u_{0}^{t, x_{0}}(y)$. Moreover, if we set $x=x_{0}+\sqrt{t} y$, we have that $\partial / \partial y^{h}=\sqrt{t} \partial / \partial x^{h}$ and also that the unit outward normal to $\partial \Omega^{t, x_{0}}$ at $y$ coincides with the unit outward normal to $\partial \Omega$ at $x$; therefore,

$$
\left\langle A^{t, x_{0}}(y) D_{y} v(s, y), v(y)\right\rangle=\sqrt{t}\left\langle A(x) D_{x} u(t s, x), v(x)\right\rangle=0 .
$$

In the same way, we have

$$
\partial_{s} v(s, y)=t u^{\prime}\left(t s, x_{0}+\sqrt{t} y\right)=t u^{\prime}(t s, x)=t \mathcal{A}(x) u(t s, x)=\mathcal{A}^{t, x_{0}}(y) v(s, y),
$$

where $u^{\prime}$ denotes the derivative of $u$ with respect to its first variable, and this concludes the proof.

We also denote by $\left(T^{t, x_{0}}(s)\right)_{s \geq 0}$ the semigroup associated with problem (6.2) and by $p^{t, x_{0}}(s, y, z)$ its kernel. We also denote by $\left(T^{x_{0}}(s)\right)_{s \geq 0}$ the semigroup associated with the problem

$$
\begin{cases}\partial_{s} w(s, y)=\mathcal{A}^{x_{0}}(y) w(s, y) & \text { in }(0,+\infty) \times \mathbb{R}^{N}, \\ w(0, y)=w_{0}(y) & \text { in } \mathbb{R}^{N}\end{cases}
$$

and by $p^{x_{0}}(s, y, z)$ its kernel.

Lemma 6.2 For the kernels the following holds

$$
p(s, x, y)=t^{-N / 2} p^{t, x_{0}}\left(\frac{s}{t}, \frac{x-x_{0}}{\sqrt{t}}, \frac{y-x_{0}}{\sqrt{t}}\right) .
$$

Proof The proof of Lemma 6.1 gives that $v(s, y)=T^{t, x_{0}}(s) u_{0}^{t, x_{0}}(y)=T(t s) u_{0}\left(x_{0}+\sqrt{t} y\right)$; using the kernels, we get that

$$
\begin{aligned}
\int_{\Omega} p(s, x, y) u_{0}(y) d y & =T(s) u_{0}(x)=T^{t, x_{0}}\left(\frac{s}{t}\right) u_{0}^{t, x_{0}}\left(\frac{x-x_{0}}{\sqrt{t}}\right) \\
& =\int_{\Omega^{t, x_{0}}} p^{t, x_{0}}\left(\frac{s}{t}, \frac{x-x_{0}}{\sqrt{t}}, z\right) u_{0}\left(x_{0}+\sqrt{t} z\right) d z \\
& =t^{-N / 2} \int_{\Omega} p^{t, x_{0}}\left(\frac{s}{t}, \frac{x-x_{0}}{\sqrt{t}}, \frac{y-x_{0}}{\sqrt{t}}\right) u_{0}(y) d y .
\end{aligned}
$$

The arbitrarity of $u_{0}$ gives the thesis.

We have the following result.

Proposition 6.3 For every $f \in L^{1}\left(\mathbb{R}^{N}\right)$, let $u^{t, x}(s, \xi)$ be the solution of the problem

$$
\begin{cases}\partial_{s} w(s, \xi)=\mathcal{A}^{t, x}(\xi) w(s, \xi) & \text { in }(0,+\infty) \times \Omega^{t, x}, \\ \left\langle A(x+\sqrt{t} \xi) D w(s, \xi), v_{\Omega^{t, x}}(\xi)\right\rangle=0 & \text { in }(0,+\infty) \times \partial \Omega^{t, x}, \\ w(0, \xi)=f(\xi) & \text { in } \Omega^{t, x}\end{cases}
$$

and let $u^{x}(s, \xi)$ be the solution of the problem

$$
\begin{cases}\partial_{s} w(s, \xi)=\mathcal{A}^{x}(\xi) w(s, \xi) & \text { in }(0,+\infty) \times \mathbb{R}^{N}, \\ w(0, \xi)=f(\xi) & \text { in } \mathbb{R}^{N} .\end{cases}
$$

Then for every $s>0$ we have that $u^{t, x}(s, \cdot)$ converges to $u^{x}(s, \cdot)$ in $L_{\text {loc }}^{1}\left(\mathbb{R}^{N}\right)$ as $t \rightarrow 0$. 
Proof We start by taking $f \in C_{c}\left(\mathbb{R}^{N}\right)$ and denote by $u^{t, x}(s, \xi)$ the solution of the problem

$$
\begin{cases}\partial_{s} w(s, \xi)=\mathcal{A}^{t, x}(\xi) w(s, \xi) & \text { in }(0,+\infty) \times \Omega^{t, x}, \\ \left\langle A^{t, x}(\xi) D_{\xi} w(s, \xi), v(\xi)\right\rangle=0 & \text { in }(0,+\infty) \times \partial \Omega^{t, x}, \\ w(0, \xi)=f(\xi) & \text { in } \Omega^{t, x} .\end{cases}
$$

Since $u^{t, x}$ is a classical solution, for every regular function $\varphi:\left[0, s_{0}\right] \times \mathbb{R}^{N} \rightarrow \mathbb{R}$ with $\varphi\left(s_{0}, \cdot\right)=0$, the following holds:

$$
\begin{aligned}
-\int_{\Omega^{t, x}} f(\xi) \varphi(0, \xi) d \xi= & \int_{0}^{s_{0}} \int_{\Omega^{t, x}}\left\{u^{t, x}(s, \xi)\left(\partial_{s} \varphi(s, \xi)+t C^{t, x}(\xi)\right)\right. \\
& \left.+\frac{\partial u^{t, x}(s, \xi)}{\partial \xi^{k}}\left[-A_{h k}^{t, x}(\xi) \frac{\partial \varphi(s, \xi)}{\partial \xi^{h}}+\sqrt{t} \varphi(s, \xi) B_{k}^{t, x}(\xi)\right]\right\} d \xi d s .
\end{aligned}
$$

Moreover, notice that $t C^{t, x} \rightarrow 0, A_{h k}^{t, x} \rightarrow A_{h k}(x), \sqrt{t} B_{k}^{t, x} \rightarrow 0$ uniformly on compact sets as $t \rightarrow 0$.

As an auxiliary tool, let us use the $L^{2}$ theory, see, e.g., [18, Sect. 5.4], recalling that there is $M>0$ such that

$$
\begin{aligned}
\left\|u^{t, x}(s)\right\|_{L^{2}\left(\Omega^{t, x}\right)} & \leq M\|f\|_{L^{2}\left(\Omega^{t, x}\right)} \leq M\|f\|_{L^{2}\left(\mathbb{R}^{N}\right)}, \\
\left\|D u^{t, x}(s)\right\|_{L^{2}\left(\Omega^{t, x}\right)} & \leq \frac{M}{\sqrt{s}}\|f\|_{L^{2}\left(\Omega^{t, x}\right)} \leq \frac{M}{\sqrt{s}}\|f\|_{L^{2}\left(\mathbb{R}^{N}\right)},
\end{aligned}
$$

and

$$
\left\|D^{2} u^{t, x}(s)\right\|_{L^{2}\left(\Omega^{t, x}\right)} \leq \frac{M}{s}\|f\|_{L^{2}\left(\Omega^{t, x}\right)} \leq \frac{M}{s}\|f\|_{L^{2}\left(\mathbb{R}^{N}\right)} .
$$

These conditions imply that for every bounded open set $K \subset \mathbb{R}^{N}, s>0$ fixed and $t_{0}$ small enough, the family $\left(u^{t, x}(s, \cdot)\right)_{0<t<t_{0}}$ is bounded in $W^{2,2}(K)$, and then, up to subsequences, it is strongly convergent in $W^{1,2}(K)$ and also in $W^{1,1}(K)$.

We can now fix a countable dense set $D \subset\left[0, s_{0}\right]$ in such a way that $u^{t_{h}, x}(s, \cdot)$ converges to some $g(s, \cdot)$ in $W^{1,1}(K)$ for every $s \in D$ and some sequence $t_{h} \rightarrow 0$. By Theorem 3.1 we get that

$$
\begin{gathered}
\left\|u^{t, x}\left(s_{2}, \cdot\right)-u^{t, x}\left(s_{1}, \cdot\right)\right\|_{L^{1}\left(\Omega^{t, x}\right)}=\left\|\int_{s_{1}}^{s_{2}} \partial_{s} u^{t, x}(s, \cdot) d s\right\|_{L^{1}\left(\Omega^{t, x}\right)} \\
\leq \int_{s_{1}}^{s_{2}}\left\|A^{t, x} u^{t, x}(s, \cdot)\right\|_{L^{1}\left(\Omega^{t, x}\right)} d s \leq c_{2}\|f\|_{L^{1}\left(\Omega^{t, x}\right)} \int_{s_{1}}^{s_{2}} \frac{1}{s} d s \leq c_{2}\|f\|_{L^{1}\left(\mathbb{R}^{N}\right)} \log \frac{s_{2}}{s_{1}},
\end{gathered}
$$

that is, the function $s \mapsto u^{t, x}(s, \cdot)$ is continuous from $\left(0, s_{0}\right)$ to $L^{1}\left(\Omega^{t, x}\right)$; in particular, if we consider $s_{1}, s_{2} \in D$, then the inequality

$$
\begin{aligned}
\left\|g\left(s_{2}, \cdot\right)-g\left(s_{1}, \cdot\right)\right\|_{L^{1}(K)} \leq & \left\|g\left(s_{2}, \cdot\right)-u^{t_{h}, x}\left(s_{2}, \cdot\right)\right\|_{L^{1}(K)}+\left\|u^{t_{h}, x}\left(s_{2}, \cdot\right)-u^{t_{h}, x}\left(s_{1}, \cdot\right)\right\|_{L^{1}(K)} \\
& +\left\|u^{t_{h}, x}\left(s_{1}, \cdot\right)-g\left(s_{1}, \cdot\right)\right\|_{L^{1}(K)}
\end{aligned}
$$

holds and the convergence of $u^{t, x}$ on $D$ shows that we can extend $g$ to a continuous map from $\left(0, s_{0}\right)$ to $L_{\text {loc }}^{1}\left(\mathbb{R}^{N}\right)$; we also notice that by (3.2) we deduce also that $g(s, \cdot) \in W^{1,1}(K)$ for every $s \in\left(0, s_{0}\right)$. By continuity, and by the convergence of $u^{t_{h}, x}(s, \cdot)$ on $D$ we deduce that 
$u^{t_{h}, x}(s, \cdot) \rightarrow g(s, \cdot)$ in $L_{\text {loc }}^{1}\left(\mathbb{R}^{N}\right)$ for every $s \in\left(0, s_{0}\right)$. In addition, conditions (3.2) allow us to apply the dominated convergence theorem, and then, taking the limit in (6.5), we get

$$
-\int_{K} f(\xi) \varphi(0, \xi) d \xi=\int_{0}^{s_{0}} \int_{K}\left(g(s, \xi) \partial_{s} \varphi(s, \xi)-\left\langle A(x) D_{\xi} \varphi(s, \xi), D_{\xi} g(s, \xi)\right\rangle\right) d \xi d s
$$

for all $\varphi$ as above, and then (see, e.g., [17, Proposition 2.1, Chap. III]) $g(s, \cdot)$ is the solution of the problem

$$
\begin{cases}\partial_{s} w(s, \xi)=A_{h k}(x) \frac{\partial^{2} w}{\partial \xi^{h} \partial \xi^{k}}(s, \xi) & \text { in }\left(0, s_{0}\right) \times \mathbb{R}^{N}, \\ w(0, \xi)=f(\xi) & \text { in } \mathbb{R}^{N}\end{cases}
$$

for every $f \in C_{c}\left(\mathbb{R}^{N}\right)$. Then, it follows that

$$
g(s, \xi)=u^{x}(s, \xi)=\int_{\mathbb{R}^{N}} p^{x}(s, \xi, z) f(z) d z,
$$

where using the Fourier transform the kernel $p^{x}$ is given by

$$
p^{x}(s, \xi, z)=\frac{1}{(4 \pi s)^{N / 2}\left|\operatorname{det} A^{1 / 2}(x)\right|} \exp \left(-\frac{\left\langle A^{-1}(x)(\xi-z),(\xi-z)\right\rangle}{4 s}\right) .
$$

From the density of $C_{c}$ in $L^{1}$ we conclude.

The following statement is an immediate consequence of Proposition 6.3.

Corollary 6.4 For every $s>0$, a.e. $\xi \in \mathbb{R}^{N}$, the measures $d \mu^{t, x}=p^{t, x}(s, \xi, \cdot) d \mathcal{L}^{N}\left\llcorner\Omega^{t, x}\right.$ are weakly* convergent to the measure $d \mu^{x}=p^{x}(s, \xi, \cdot) d \mathcal{L}^{N}$ as $t \rightarrow 0$, that is, for every $\varphi \in C_{c}\left(\mathbb{R}^{N}\right)$ the following equality holds

$$
\lim _{t \rightarrow 0} \int_{\Omega^{t, x}} \varphi(z) p^{t, x}(s, \xi, z) d z=\int_{\mathbb{R}^{N}} \varphi(z) p^{x}(s, \xi, z) d z .
$$

Henceforth, given the function $p(s, \xi, z)$, we shall denote by $D_{1} p(s, \xi, z)$ the gradient with respect to the first spatial variables $\xi$ and by $D_{2} p(s, \xi, z)$ the gradient with respect to the second spatial variables $z$.

Proposition 6.5 For every $s>0$ and every a.e. $\xi \in \mathbb{R}^{N}$, the equality

$$
\lim _{t \rightarrow 0} \int_{\Omega^{t, x}}\left\langle D_{2} p^{t, x}(s, \xi, z), \varphi(z)\right\rangle d z=\int_{\mathbb{R}^{N}}\left\langle D_{2} p^{x}(s, \xi, z), \varphi(z)\right\rangle d z
$$

holds for every $\varphi \in C_{c}\left(\mathbb{R}^{N}, \mathbb{R}^{N}\right)$.

Proof We start by considering $\varphi \in C_{c}^{1}\left(\mathbb{R}^{N}, \mathbb{R}^{N}\right)$; we choose $t_{0}>0$ in such a way that $\operatorname{supp} \varphi \subset \Omega^{t, x}$ for all $t \leq t_{0}$; then

$$
\int_{\Omega^{t, x}}\left\langle D_{2} p^{t, x}(s, \xi, z), \varphi(z)\right\rangle d z=-\int_{\Omega^{t, x}} p^{t, x}(s, \xi, z) \operatorname{div} \varphi(z) d z
$$


and then, by Corollary 6.4

$$
\begin{aligned}
\lim _{t \rightarrow 0} \int_{\Omega^{t, x}}\left\langle D_{2} p^{t, x}(s, \xi, z), \varphi(z)\right\rangle d z=\lim _{t \rightarrow 0}-\int_{\Omega^{t, x}} p^{t, x}(s, \xi, z) \operatorname{div} \varphi(z) d z \\
\quad=-\int_{\mathbb{R}^{N}} p^{x}(s, \xi, z) \operatorname{div} \varphi(z) d z=\int_{\mathbb{R}^{N}}\left\langle D_{2} p^{x}(s, \xi, z), \varphi(z)\right\rangle d z .
\end{aligned}
$$

For an arbitrary $\varphi \in C_{c}\left(\mathbb{R}^{N}, \mathbb{R}^{N}\right)$ we use an approximation procedure; we select $\varphi_{\varepsilon} \in$ $C_{c}^{1}\left(\mathbb{R}^{N}, \mathbb{R}^{N}\right)$ such that $\left\|\varphi-\varphi_{\varepsilon}\right\|_{\infty} \leq \varepsilon$ and then

$$
\begin{aligned}
\int_{\Omega^{t, x}}\left\langle D_{2} p^{t, x}(s, \xi, z), \varphi(z)\right\rangle d z= & \int_{\Omega^{t, x}}\left\langle D_{2} p^{t, x}(s, \xi, z), \varphi_{\varepsilon}(z)\right\rangle d z \\
& +\int_{\Omega^{t, x}}\left\langle D_{2} p^{t, x}(s, \xi, z),\left(\varphi(z)-\varphi_{\varepsilon}(z)\right)\right\rangle d z .
\end{aligned}
$$

Taking into account that $p^{t, x}(s, \xi, z)=t^{N / 2} p(t s, x+\sqrt{t} \xi, x+\sqrt{t} z)$ and also that

$$
\begin{aligned}
D_{2} p^{t, x}(s, \xi, z) & =D_{z} t^{N / 2} p(t s, x+\sqrt{t} \xi, x+\sqrt{t} z) \\
& =t^{(N+1) / 2} D_{2} p(t s, x+\sqrt{t} \xi, x+\sqrt{t} z)
\end{aligned}
$$

by (3.1) we obtain

$$
\begin{aligned}
& \left|\int_{\Omega^{t, x}}\left\langle D_{2} p^{t, x}(s, \xi, z),\left(\varphi(z)-\varphi_{\varepsilon}(z)\right)\right\rangle d z\right| \\
& \quad \leq t^{(N+1) / 2}\left\|\varphi-\varphi_{\varepsilon}\right\|_{\infty} \int_{\Omega^{t, x}}\left|D_{2} p(t s, x+\sqrt{t} \xi, x+\sqrt{t} z)\right| d z \leq C \varepsilon
\end{aligned}
$$

with $C$ independent of $t$. Of course, the inequality

$$
\left|\int_{\mathbb{R}^{N}}\left\langle D_{2} p^{x}(s, \xi, z),\left(\varphi(z)-\varphi_{\varepsilon}(z)\right)\right\rangle d z\right| \leq C \varepsilon
$$

holds as well, and then

$$
\begin{aligned}
& \lim _{t \rightarrow 0}\left|\int_{\Omega^{t, x}}\left\langle D_{2} p^{t, x}(s, \xi, z), \varphi(z)\right\rangle d z-\int_{\mathbb{R}^{N}}\left\langle D_{2} p^{x}(s, \xi, z), \varphi(z)\right\rangle d z\right| \\
& \quad \leq \lim _{t \rightarrow 0}\left|\int_{\Omega^{t, x}}\left\langle D_{2} p^{t, x}(s, \xi, z),\left(\varphi(z)-\varphi_{\varepsilon}(z)\right)\right\rangle d z\right|
\end{aligned}
$$




$$
\begin{aligned}
& +\lim _{t \rightarrow 0}\left|\int_{\Omega^{t, x}}\left\langle D_{2} p^{t, x}(s, \xi, z), \varphi_{\varepsilon}(z)\right\rangle d z-\int_{\mathbb{R}^{N}}\left\langle D_{2} p^{x}(s, \xi, z), \varphi_{\varepsilon}(z) d z\right\rangle\right| \\
& +\lim _{t \rightarrow 0}\left|\int_{\mathbb{R}^{N}}\left\langle D_{2} p^{x}(s, \xi, z),\left(\varphi(z)-\varphi_{\varepsilon}(z)\right)\right\rangle d z\right| \leq C \varepsilon
\end{aligned}
$$

and the thesis follows from the arbitrariness of $\varepsilon$.

The main step in the proof of (1.5) is the following result, where an asymptotic formula relating two sets of finite perimeter is shown. In the statement, we assume that $E$ has finite measure in order to give a meaning to the left hand side in (6.11). But, notice that, since $E$ has finite perimeter in $\Omega$, then by the relative isoperimetric inequality in the regular set $\Omega$

$$
\min \{|E \cap \Omega|,|\Omega \backslash E|\} \leq c P(E, \Omega)^{N / N-1},
$$

either $|E \cap \Omega|$ or $|\Omega \backslash E|$ is finite. Therefore, if $|E \cap \Omega|$ is infinite, then $|\Omega \backslash E|$ is finite and (6.11) applies with $\Omega \backslash E$ in place of $E$.

Theorem 6.6 Assume (H1), (H2), (H3), and let $(T(t))_{t \geq 0}$ be the semigroup generated by $(\mathcal{A}, D(\mathcal{A}))$ in $L^{1}(\Omega)$; then, if $E, F \subset \mathbb{R}^{N}$ are sets of finite perimeter in $\Omega$, the following holds

$$
\lim _{t \rightarrow 0} \sqrt{\frac{\pi}{t}} \int_{\Omega \cap F}\left(\chi_{E}(x)-T(t) \chi_{E}(x)\right) d x=\int_{\Omega \cap \mathcal{F} F \cap \mathcal{F} E}\left\langle A(x) v_{E}(x), v_{F}(x)\right\rangle d \mathcal{H}^{N-1}(x) .
$$

Proof We have

$$
\begin{aligned}
& \int_{\Omega \cap F}\left(T(t) \chi_{E}(x)-\chi_{E}(x)\right) d x=\int_{\Omega \cap F} \int_{0}^{t} \frac{d}{d s} T(s) \chi_{E}(x) d s d x \\
& =\int_{0}^{t} \int_{\Omega \cap F} \mathcal{A} T(s) \chi_{E}(x) d x d s=\int_{0}^{t}\left(\int_{\Omega \cap F} \operatorname{div}_{x}\left(A(x) D_{x} T(s) \chi_{E}(x)\right) d x\right. \\
& \left.\quad+\int_{\Omega \cap F}\left\langle B(x), D_{x} T(s) \chi_{E}(x)\right\rangle d x+\int_{\Omega \cap F} C(x) T(s) \chi_{E}(x) d x\right) d s .
\end{aligned}
$$

For the last term we have that

$$
\left|\int_{\Omega \cap F} C(x) T(s) \chi_{E}(x) d x\right| \leq\|C\|_{\infty} \min \{|\Omega \cap E|,,|\Omega \cap F|\}
$$

and then

$$
\lim _{t \rightarrow 0} \frac{1}{\sqrt{t}} \int_{0}^{t} \int_{\Omega \cap F} C(x) T(s) \chi_{E}(x) d x d s=0
$$


For the second term in (6.12), we notice that

$\left|\int_{\Omega \cap F} \int_{\Omega \cap E}\left\langle B(x), D_{x} p(s, x, y)\right\rangle d y d x\right| \leq\|B\|_{\infty} \min \{|\Omega \cap E|,|\Omega \cap F|\} \int_{\Omega}\left|D_{x} p(s, x, y)\right| d x$

and using Gaussian estimates (3.1) we get

$$
\int_{\Omega}\left|D_{x} p(s, x, y)\right| d x \leq \frac{c}{\sqrt{s}}
$$

for some constant $c$ depending only on the operator $\mathcal{A}$ and the dimension $N$. We introduce now the kernel $p_{*}(s, x, y)$ of the semigroup generated by the adjoint operator $\mathcal{A}^{*}$ of $\mathcal{A}$; by the symmetry of the matrix $A$, the second order part of $\mathcal{A}^{*}$ is the same as $\mathcal{A}$. In this way we have that $p(s, x, y)=p_{*}(s, y, x)$ (see for instance [18, Theorem 5.6]) and since

$$
\begin{gathered}
\frac{\partial}{\partial x^{i}} p(s, x, y)=\lim _{h \rightarrow 0} \frac{p\left(s, x+h e_{i}, y\right)-p(s, x, y)}{h}=\lim _{h \rightarrow 0} \frac{p_{*}\left(s, y, x+h e_{i}\right)-p_{*}(s, y, x)}{h} \\
=s^{-N / 2} \lim _{h \rightarrow 0} \frac{p_{*}^{s, x}\left(1, \frac{y-x}{\sqrt{s}}, \frac{h e_{i}}{\sqrt{s}}\right)-p_{*}^{s, x}\left(1, \frac{y-x}{\sqrt{s}}, 0\right)}{h}=s^{-(N+1) / 2} D_{2}^{i} p_{*}^{s, x}\left(1, \frac{y-x}{\sqrt{s}}, 0\right)
\end{gathered}
$$

where $D_{2}^{i}$ denotes the $i$ th component of the gradient with respect to the second variables. Then $D_{x} p(s, x, y)=s^{-(N+1) / 2} D_{2} p_{*}^{s, x}\left(1, \frac{y-x}{\sqrt{s}}, 0\right)$; hence

$$
\begin{aligned}
\int_{\Omega \cap F}\left\langle B, D_{x} T(s) \chi_{E}\right\rangle d x & =\int_{\Omega \cap F} d x \int_{\Omega \cap E}\left\langle B(x), D_{x} p(s, x, y)\right\rangle d y \\
& =s^{-(N+1) / 2} \int_{\Omega \cap F} d x \int_{\Omega \cap E}\left\langle B(x), D_{2} p_{*}^{s, x}\left(1, \frac{y-x}{\sqrt{s}}, 0\right)\right\rangle d y \\
& =\frac{1}{\sqrt{s}} \int_{\Omega \cap F} d x \int_{\Omega^{s, x} \cap E^{s, x}}\left\langle B(x), D_{2} p_{*}^{s, x}(1, z, 0)\right\rangle d z \\
& =\frac{1}{\sqrt{s}} \int_{\Omega \cap F} d x \int_{\mathbb{R}^{N}}\left\langle B(x), D_{2} p_{*}^{s, x}(1, z, 0)\right\rangle d \mu^{s, x}(z),
\end{aligned}
$$

where we have denoted by $\mu^{s, x}$ the measure

$$
\mu^{s, x}=\mathcal{L}^{N}\left\llcorner\left(\Omega^{s, x} \cap E^{s, x}\right) .\right.
$$

These measures verify the following properties:

1. $\mu^{s, x} \rightarrow^{*} 0$ if $x \in E^{0}$;

2. $\mu^{s, x} \rightarrow^{*} \mathcal{L}^{N}$ if $x \in E^{1}$;

3. $\mu^{s, x} \rightarrow^{*} \mathcal{L}^{N}\left\llcorner H_{v_{E}(x)}\right.$ for $x \in \mathcal{F} E$, where $H_{v_{E}(x)}=\left\{z \in \mathbb{R}^{N}:\left\langle z, v_{E}(x)\right\rangle \leq 0\right\}$.

These facts imply that, for $x \in E^{0}, \int_{\mathbb{R}^{N}}\left\langle B(x), D_{2} p_{*}^{s, x}(1, z, 0)\right\rangle d \mu^{s, x}(z) \rightarrow 0$; moreover, for $x \in E^{1}$

$$
\int_{\mathbb{R}^{N}}\left\langle B(x), D_{2} p_{*}^{s, x}(1, z, 0)\right\rangle d \mu^{s, x}(z) \rightarrow B(x) \cdot \int_{\mathbb{R}^{N}} D_{2} p_{*}^{x}(1, z, 0) d z=0 .
$$


Taking into account that $\left|F \backslash\left(E^{0} \cup E^{1}\right)\right|=0$ and the dominated convergence Theorem, we have then obtained that

$$
\lim _{t \rightarrow 0} \frac{1}{\sqrt{t}} \int_{0}^{t} \int_{\Omega \cap F} \int_{\Omega \cap E}\left\langle B(x), D_{x} p(s, x, y)\right\rangle d y d x d s=0 .
$$

It remains to study the first term of (6.12); with an integration by parts and recalling that $\mathcal{H}^{N-1}\left(\partial^{*} E \backslash \mathcal{F} E\right)=0$, we get

$$
\begin{aligned}
& \int_{\Omega \cap F} \operatorname{div}\left(A D_{x} T(s) \chi_{E}(x)\right) d x=\int_{\Omega \cap \mathcal{F} F}\left\langle D_{x} T(s) \chi_{E}(x), A(x) v_{F}(x)\right\rangle d \mathcal{H}^{N-1}(x) \\
& =\int_{\Omega \cap \mathcal{F} F} \int_{\Omega \cap E} s^{-(N+1) / 2}\left\langle D_{2} p_{*}^{s, x}\left(1, \frac{y-x}{\sqrt{s}}, 0\right), A(x) v_{F}(x)\right\rangle d y d \mathcal{H}^{N-1}(x) \\
& =-\frac{1}{\sqrt{s}} \int_{\Omega \cap \mathcal{F} F} \int_{\Omega^{s, x} \cap E^{s, x}}\left\langle D_{2} p_{*}^{s, x}(1, z, 0), A(x) v_{F}(x)\right\rangle d z d \mathcal{H}^{N-1}(x) \\
& =-\frac{1}{\sqrt{s}} \int_{\Omega \cap \mathcal{F} F} \int_{\mathbb{R}^{N}}\left\langle D_{2} p_{*}^{s, x}(1, z, 0), A(x) v_{F}(x)\right\rangle d \mu^{s, x}(z) d \mathcal{H}^{N-1}(x) .
\end{aligned}
$$

With the same argument previously used, we can deduce that for $x \in E^{0} \cup E^{1}$, the limit of the above integral as $t \rightarrow 0$ vanishes; then we can only consider points $x \in \mathcal{F} F \cap \mathcal{F} E$; in this case we obtain that

$$
\int_{\mathbb{R}^{N}}\left\langle D_{2} p_{*}^{s, x}(1, z, 0), A(x) v_{F}(x)\right\rangle d \mu^{s, x}(z) \longrightarrow \int_{H_{v_{E}}(x)}\left\langle D_{2} p_{*}^{x}(1, z, 0), A(x) v_{F}(x)\right\rangle d z .
$$

Taking into account (6.9) and the symmetry of $A$, we get that

$$
D_{2} p_{*}^{x}(1, z, 0)=-\frac{1}{2(4 \pi)^{N / 2}\left|\operatorname{det} A^{1 / 2}(x)\right|} \exp \left(-\left\langle A^{-1}(x) z, z\right\rangle / 4\right) A^{-1}(x) z
$$

and then, since for $x \in \mathcal{F} F \cap \mathcal{F} E$ we have $v_{F}(x)=\left\langle v_{E}(x), v_{F}(x)\right\rangle v_{E}(x)$

$$
\begin{aligned}
& \int_{H_{\nu_{E}(x)}}\left\langle D_{2} p_{*}^{x}(1, z, 0), A(x) v_{F}(x)\right\rangle d z \\
& =-\frac{\left\langle v_{E}(x), v_{F}(x)\right\rangle}{2(4 \pi)^{N / 2}\left|\operatorname{det} A^{1 / 2}(x)\right|} \int_{H_{v_{E}}(x)} \exp \left(-\left\langle A^{-1}(x) z, z\right) / 4\right)\left\langle z, v_{E}(x)\right\rangle d z \\
& =-\frac{\left\langle v_{E}(x), v_{F}(x)\right\rangle}{\pi^{N / 2}} \int_{H_{A^{1 / 2}(x) v_{E}(x)}} e^{-|z|^{2}}\left\langle z, A^{1 / 2}(x) v_{E}(x)\right\rangle d z .
\end{aligned}
$$

For the computation of this last integral, we consider an orthonormal basis $\left\{e_{1}, \ldots, e_{N}\right\}$ of $\mathbb{R}^{N}$ with

$$
e_{N}=\frac{A^{1 / 2}(x) v_{E}(x)}{\left|A^{1 / 2}(x) v_{E}(x)\right|}
$$


we then obtain

$$
\begin{aligned}
& \int_{H_{A^{1 / 2}(x) v_{E}(x)}}\left\langle z, A^{1 / 2}(x) v_{E}(x)\left|e^{-|z|^{2}} d z=\right| A^{1 / 2}(x) v_{E}(x)\right| \int_{H_{A^{1 / 2}(x) v_{E}(x)}} z_{N} e^{-|z|^{2}} d z \\
& =\pi^{(N-1) / 2}\left|A^{1 / 2}(x) v_{E}(x)\right| \int_{-\infty}^{0} z_{N} e^{-z_{N}^{2}} d z_{N}=-\frac{\pi^{(N-1) / 2}}{2}\left|A^{1 / 2}(x) v_{E}(x)\right| .
\end{aligned}
$$

At the end, we have obtained that

$$
\lim _{t \rightarrow 0} \sqrt{\frac{\pi}{t}} \int_{\Omega \cap F}\left(T(t) \chi_{E}-\chi_{E}\right) d x=-\int_{\Omega \cap \mathcal{F} F \cap \mathcal{F} E}\left\langle v_{E}, v_{F}\right\rangle\left|A^{1 / 2} v_{E}\right| d \mathcal{H}^{N-1} .
$$

Specializing the above result for $F=E^{c}$ we get the following

Corollary 6.7 Assume $(\mathbf{H 1}),(\mathbf{H 2}),(\mathrm{H} 3)$, and let $(T(t))_{t \geq 0}$ be the semigroup generated by $\left(\mathcal{A}, D(\mathcal{A})\right.$ ) in $L^{1}(\Omega)$; then, if $E \subset \mathbb{R}^{N}$ is a set with finite perimeter in $\Omega$, the following equality holds:

$$
\lim _{t \rightarrow 0} \sqrt{\frac{\pi}{t}} \int_{\Omega \cap E^{c}} T(t) \chi_{E} d x=\int_{\Omega \cap \mathcal{F} E}\left|A^{1 / 2}(x) v_{E}(x)\right| d \mathcal{H}^{N-1}(x) .
$$

Remark 6.8 Using an argument similar to the one used in [15, Theorem 3.4], it is possible to prove that if $E$ is a set with finite measure such that

$$
\liminf _{t \rightarrow 0} \frac{1}{\sqrt{t}} \int_{E^{c} \cap \Omega} T(t) \chi_{E}(x) d x<+\infty,
$$

then $E$ has finite perimeter in $\Omega$, that is $\chi_{E} \in B V(\Omega)$.

We are now in a position to prove the main result of this section, namely, the announced characterization of $B V$ functions (1.5). The strategy is the same as for $\mathbb{R}^{N}$, see [15], and is based on (4.2).

Theorem 6.9 Assume (H1), (H2), (H3), and let $(T(t))_{t \geq 0}$ be the semigroup generated by $(\mathcal{A}, D(\mathcal{A}))$ in $L^{1}(\Omega)$ and let $u \in L^{1}(\Omega)$; then $u \in B V(\Omega)$ if and only if

$$
\liminf _{t \rightarrow 0} \frac{1}{\sqrt{t}} \int_{\Omega \times \Omega}|u(x)-u(y)| p(t, x, y) d x d y<+\infty
$$

moreover, in this case the following equality holds

$$
|D u|_{A}(\Omega)=\lim _{t \rightarrow 0} \frac{\sqrt{\pi}}{2 \sqrt{t}} \int_{\Omega \times \Omega}|u(x)-u(y)| p(t, x, y) d x d y .
$$


Proof We start by considering $u \in L^{1}(\Omega)$; for $\tau \in \mathbb{R}$ we denote by $E_{\tau}=\{u>\tau\}$ and, since the semigroup is positive and contractive, we obtain that

$$
\begin{aligned}
0 & \leq \int_{\mathbb{R}} \liminf _{t \rightarrow 0} \frac{1}{\sqrt{t}} \int_{E_{\tau}^{c} \cap \Omega} T(t) \chi_{E_{\tau}} d x d \tau \leq \liminf _{t \rightarrow 0} \frac{1}{\sqrt{t}} \int_{\mathbb{R}} \int_{E_{\tau}^{c} \cap \Omega} T(t) \chi_{E_{\tau}} d x d \tau \\
& \leq \liminf _{t \rightarrow 0} \frac{1}{\sqrt{t}} \int_{\Omega \times \Omega} \int_{\mathbb{R}}\left|\chi_{E_{\tau}}(x)-\chi_{E_{\tau}}(y)\right| p(t, x, y) d x d y d \tau \\
& =\liminf _{t \rightarrow 0} \frac{1}{\sqrt{t}} \int_{\Omega \times \Omega}|u(x)-u(y)| p(t, x, y) d x d y<+\infty
\end{aligned}
$$

and then, thanks to Remark 6.8, almost every level $E_{\tau}$ has finite perimeter and equation (6.14) holds. Then, using coarea formula (4.2), we get

$$
\begin{aligned}
|D u|_{A}(\Omega) & =\int_{\mathbb{R}} P_{A}\left(E_{\tau}, \Omega\right) d \tau=\int_{\mathbb{R}} \lim _{t \rightarrow 0} \sqrt{\frac{\pi}{t}} \int_{E_{\tau}^{c \cap \Omega}} T(t) \chi_{E_{\tau}} d x d \tau \\
& \leq \liminf _{t \rightarrow 0} \sqrt{\frac{\pi}{t}} \int_{\Omega \times \Omega}|u(x)-u(y)| p(t, x, y) d x d y<+\infty
\end{aligned}
$$

that is $u \in B V_{A}(\Omega)$. The other implication follows from (6.15). To prove (6.15), we define the function

$$
g_{t}(\tau)=\sqrt{\frac{\pi}{t}} \int_{E_{\tau}^{c \cap} \Omega} T(t) \chi_{E_{\tau}}(x) d x .
$$

For this function we have the following estimate

$$
\begin{aligned}
\left|g_{t}(\tau)\right|= & \sqrt{\frac{\pi}{t}}\left|\int_{0}^{t} \int_{E_{\tau}^{c} \cap \Omega} \mathcal{A} T(s) \chi_{E_{\tau}} d x d s\right|=\sqrt{\frac{\pi}{t}} \mid \int_{0}^{t}\left(\int_{\mathcal{F} E_{\tau} \cap \Omega}\left\langle A D T(s) \chi_{E_{\tau}}, v_{E_{\tau}}\right) d \mathcal{H}^{N-1}\right. \\
& \left.\left.+\int_{E_{\tau}^{c \cap \Omega}}\left\langle B, D T(s) \chi_{E_{\tau}}\right\rangle d x+\int_{E_{\tau}^{c} \cap \Omega} C T(s) \chi_{E_{\tau}}\right) d x\right) d s \mid \\
\leq & \sqrt{\frac{\pi}{t}} \int_{0}^{t}\left(\|A\|_{\infty} \int_{\mathcal{F} E_{\tau}}\left|D T(s) \chi_{E_{\tau}}\right| d \mathcal{H}^{N-1}\right. \\
& \left.+\|B\|_{\infty} \int_{E_{\tau}^{c} \cap \Omega}\left|D_{E_{\tau} \cap \Omega} p(s, x, y)\right| d x d y+\|C\|_{\infty} \int_{E_{\tau}^{c} \cap \Omega} \underset{E_{\tau} \cap \Omega}{\int}|p(s, x, y)| d x d y\right) d s \\
\leq & c M_{0}\left(P\left(E_{\tau}, \Omega\right)+\min \left\{\left|E_{\tau} \cap \Omega\right|,\left|E_{\tau}^{c} \cap \Omega\right|\right\}\right)=h(\tau),
\end{aligned}
$$

where the last inequality follows from the estimates (3.1) on the kernel $p(s, x, y)$. We have that $h \in L^{1}(\mathbb{R})$ since

$$
\int_{\mathbb{R}} P\left(E_{\tau}, \Omega\right) d \tau=|D u|(\Omega)
$$


and, denoted by $u^{+}=\max \{u, 0\}$ and $u^{-}=\max \{-u, 0\}$,

$$
\begin{aligned}
\int_{\mathbb{R}} \min \left\{\left|E_{\tau} \cap \Omega\right|,\left|E_{\tau}^{c} \cap \Omega\right|\right\} d \tau & \leq \int_{0}^{\infty}\left|E_{\tau} \cap \Omega\right| d \tau+\int_{-\infty}^{0}\left|E_{\tau}^{c} \cap \Omega\right| d \tau \\
& =\int_{0}^{\infty} \int_{\Omega} \chi_{E_{\tau}} d x d \tau+\int_{-\infty}^{0} \int_{\Omega} \chi_{E_{\tau}^{c}} d x d \tau \\
& =\int_{\Omega}^{\infty} \int_{0}^{\infty} \chi_{\{u>\tau\}} d \tau d x+\int_{\Omega}^{\infty} \int_{0}^{\infty} \chi_{\{-u \geq \tau\}} d \tau d x \\
& =\int_{\Omega} u^{+} d x+\int_{\Omega} u^{-} d x=\int_{\Omega}|u| d x .
\end{aligned}
$$

Then we can apply Corollary 6.7 and Lebesgue dominated convergence theorem to the functions $g_{t}$ in order to obtain

$$
\begin{aligned}
|D u|_{A}(\Omega) & =\int_{\mathbb{R}} P_{A}\left(E_{\tau}, \Omega\right) d \tau=\int_{\mathbb{R}} \lim _{t \rightarrow 0} \sqrt{\frac{\pi}{t}} \int_{E_{\tau}^{c} \cap \Omega} T(t) \chi_{E_{\tau}} d x \\
& =\lim _{t \rightarrow 0} \sqrt{\frac{\pi}{t}} \int_{\mathbb{R}} \int_{\Omega \times \Omega}\left(\chi_{E_{\tau}}(y)-\chi_{E_{\tau}}(y) \chi_{E_{\tau}}(x)\right) p(t, x, y) d x d y d \tau \\
& =\lim _{t \rightarrow 0} \sqrt{\frac{\pi}{t}} \int_{\Omega \times \Omega}(u(y)-\min \{u(y), u(x)\}) p(t, x, y) d x d y
\end{aligned}
$$

since $\chi_{E_{\tau}}(y) \chi_{E_{\tau}}(x) \neq 0$ iff $\tau<\min \{u(x), u(y)\}$; finally, the assertion follows by noticing that $\min \{u(y), u(x)\}=\frac{1}{2}(u(x)+u(y)-|u(x)-u(y)|)$.

Acknowledgments We thank Giorgio Metafune for useful discussions and suggestions.

\section{References}

1. Adams, R.A.: Sobolev Spaces. 2nd edn. Academic Press, New York (2003)

2. Ambrosio, L., Fusco, N., Pallara, D.: Functions of Bounded Variation and Free Discontinuity Problems. Oxford University Press, New York (2000)

3. Baldi, A.: Weighted BV functions. Houston J. Math. 27(3), 683-705 (2001)

4. Brézis, H.: How to recognize constant functions. Connections with Sobolev spaces. Russ. Math. Surv. 57, 693-708 (2002)

5. Butzer, P.L., Berens, H.: Semi-groups of Operators and Approximation. Springer, Berlin (1967)

6. Carbonaro, A., Mauceri, G.: A note on bounded variation and heat semigroup on Riemannian manifolds. Bull. Austral. Math. Soc. 76, 155-160 (2007)

7. Dal Maso, G.: Integral representation on $B V(\Omega)$ of $\Gamma$-limits of variational integrals. Manuscripta Math. 30(4), 387-416 (1980)

8. Dávila, J.D.: On an open question about functions of bounded variation. Calc. Var. 15, 519-527 (2002)

9. DeGiorgi, E.: Su una teoria generale della misura $(r-1)$-dimensionale in uno spazio ad $r$ dimensioni. Ann. Mat. Pura Appl. 36(4), 191-213 (1954), and also Ennio DeGiorgi: Selected Papers, (L. Ambrosio, G. Dal Maso, M. Forti, M. Miranda, S.Spagnolo eds.) Springer, Berlin (2006), 79-99. English translation, Ibid. $58-78$

10. DiBlasio, G.: Analytic semigroups generated by elliptic operators in $L^{1}$ and parabolic equations. Osaka J. Math. 28(2), 367-384 (1991) 
11. Ghisi, M., Gobbino, M.: Gradient estimates for the Perona-Malik equation. Math. Ann. 337, 557-590 (2007)

12. Henry, D.: Geometric Theory of Semilinear Parabolic Equations. Lecture Notes in Mathematics, vol. 840. Springer, Berlin (1981)

13. Ledoux, M.: Semigroup proofs of the isoperimetric inequality in Euclidean and Gauss space. Bull. Sci. Math. 118, 485-510 (1994)

14. Lunardi, A.: Analytic Semigroups and Optimal Regularity in Parabolic Problems. Birkhäuser, Boston (1995)

15. Miranda, M. Jr., Pallara, D., Paronetto, F., Preunkert, M.: Short-time heat flow and functions of bounded variation in $\mathbb{R}^{N}$. Ann. Fac. Sci. Toulouse Math. 16(1), 125-145 (2007)

16. Miranda, M. Jr., Pallara, D., Paronetto, F., Preunkert, M.: Heat semigroup and functions of bounded variation on Riemannian manifolds. J. Reine Ang. Math. 613 (2007)

17. Showalter, R.E.: Monotone Operators in Banach Space and Nonlinear Partial Differential Equations. Mathematical Surveys and Monographs 49, American Mathematical Society, Providence, RI (1997)

18. Tanabe, H.: Functional Analytic Methods in Partial Differential Equations. Marcel Dekker, New York (1997) 